\title{
High-resolution dark matter density profiles of THINGS dwarf galaxies: Correcting for non-circular motions
}

\author{
Se-Heon Oh${ }^{1}$, W.J.G. de Blok ${ }^{2}$, Fabian Walter ${ }^{3}$, Elias Brinks ${ }^{4}$, and Robert C. Kennicutt, Jr. ${ }^{5}$ \\ seheon@mso.anu.edu.au \\ edeblok@ast.uct.ac.za \\ walter@mpia.de \\ E.Brinks@herts.ac.uk \\ robk@ast.cam.ac.uk
}

\begin{abstract}
We present a new method to remove the impact of random and small-scale noncircular motions from HI velocity fields in (dwarf) galaxies in order to better constrain the dark matter properties for these objects. This method extracts the circularly rotating velocity components from the HI data cube and condenses them into a so-called bulk velocity field. We derive high-resolution $(\sim 0.2 \mathrm{kpc})$ rotation curves of IC 2574 and NGC 2366 based on bulk velocity fields derived from The HI Nearby Galaxy Survey (THINGS) obtained at the VLA. We compare the bulk velocity field rotation curves with those derived from the traditional intensity-weighted mean velocity fields and find significant differences. The bulk velocity field rotation curves are significantly less affected by non-circular motions and constrain the dark matter distribution in our galaxies, allowing us to address the discrepancy between the inferred and predicted dark matter distribution in galaxies (the "cusp/core" problem). Spitzer Infrared Nearby Galaxies Survey (SINGS) $3.6 \mu \mathrm{m}$ data, which are largely unaffected by dust in these systems, as well as ancillary optical information, are used to separate the contribution of the baryons from the total matter content. Using stellar population synthesis models, assuming various sets of metallicity and star formation histories, we compute stellar mass-to-light
\end{abstract}

\footnotetext{
${ }^{1}$ Research School of Astronomy \& Astrophysics, The Australian National University, Mount Stromlo Observatory, Cotter Road, Weston Creek, ACT 2611, Australia

${ }^{2}$ Department of Astronomy, University of Cape Town, Private Bag X3, Rondebosch 7701, South Africa

${ }^{3}$ Max-Planck-Institut für Astronomie, Königstuhl 17, 69117 Heidelberg, Germany

${ }^{4}$ Centre for Astrophysics Research, University of Hertfordshire, College Lane, Hatfield, AL10 9AB, United Kingdom

${ }^{5}$ Institute of Astronomy, University of Cambridge, Madingley Road, Cambridge CB3 0HA, United Kingdom
} 
ratios for the $3.6 \mu \mathrm{m}$ and $4.5 \mu \mathrm{m}$ bands. Using our predicted value for the $3.6 \mu \mathrm{m}$ stellar mass-to-light ratio, we find that the observed dark matter distributions of IC 2574 and NGC 2366 are inconsistent with the cuspy dark matter halo predicted by $\Lambda$ Cold Dark Matter models, even after corrections for non-circular motions. This result also holds for other assumptions about the stellar mass-to-light ratio. The distribution of dark matter within our sample galaxies is best described by models with a kpc-sized constant-density core.

Subject headings: Galaxies: dark matter - galaxies: kinematics and dynamics - galaxies: halos - galaxies (individual): IC 2574, NGC 2366

\section{Introduction}

Cosmological Cold Dark Matter (CDM) simulations have been very successful in describing the observed large-scale structures in the universe (Spergel et al. 2003; Primack 2003). They have,

however, been less successful in describing the observed dark matter density profiles of galaxies at small radii. The most commonly used models (Navarro, Frenk \& White 1996, hereafter NFW; see also Moore et al. 1998) predict that the dark matter density profile increases towards the center as a power law $\rho \sim R^{\alpha}$ with $\alpha \sim-1$ to -1.5 , giving rise to a "cusp" feature in the centers of galaxies. However, most observations do not confirm this NFW profile, instead preferring a sizeable central constant density-core with $\alpha \simeq-0.2 \pm 0.2$ (de Blok et al. 2001; de Blok \& Bosma 2002). The "cusp/core" problem is one aspect of the small-scale crisis in $\Lambda$ Cold Dark Matter $(\Lambda \mathrm{CDM})$ cosmology; the other two being the missing dwarf galaxies and the angular momentum problem (Klypin et al. 1999; Moore et al. 1999; Navarro et al. 1995).

Considerable research has been devoted to this problem, and the apparent inability of standard $\Lambda \mathrm{CDM}$ simulations to produce dark matter density profiles that match the observed profiles is now well-known (Flores \& Primack 1994; Moore 1994; de Blok et al. 2001; de Blok \& Bosma 2002; Weldrake et al. 2003; Simon et al. 2003; Gentile et al. 2004; see also Swaters et al. 2003).

It has been argued that several observational systematic effects, such as beam smearing, pointing offsets, and non-circular motions, could affect the measured inner slope of the density profile, and may therefore "hide" the signatures of cusps in the central parts of galaxies (van den Bosch et al. 2000; Swaters et al. 2003; Hayashi \& Navarro 2006). The early rotation curves used to study the dark matter density profiles in Low Surface Brightness (LSB) galaxies were mainly based on HI data with large beam-sizes, and beam-smearing could potentially have flattened the observed slopes of the rotation curves. However, high-resolution $\mathrm{H} \alpha$ rotation curves show consistency with the Hi rotation curves (de Blok et al. 2001; McGaugh et al. 2001; Kuzio de Narray et al. 2006). From this, it seems that beam smearing effects are not significant enough to erase the signature of the cusp. 
When using one-dimensional long-slit spectra, potential cusps may also be "hidden" by telescope pointing offsets (i.e., slit offsets) with respect to the galaxy centers. However, de Blok et al. (2003) show that rotation curves observed by independent observers on different telescopes agree within the given errors and that pointing offsets are unlikely to exceed $\sim 0.3^{\prime \prime}$ (see also Marchesini et al. 2002; de Blok \& Bosma 2002; de Blok 2004; Gentile et al. 2007). The insignificance of beam smearing and pointing offsets is also confirmed by high-resolution 2D optical velocity fields of LSB galaxies (Kuzio de Narray et al. 2006).

One of the fundamental assumptions of most rotation curve studies is that the tracers that are typically used for galaxy dynamics, such as $\mathrm{HI}$ or $\mathrm{H} \alpha$, travel on circular orbits. Therefore, any significant non-circularity or random motions will affect the results derived by such studies. Non-circular motions are not only thought to be due to star formation processes, but also due to bars, spiral density waves and non-circular halo potentials. For one dwarf LSB galaxy (DDO 47), Gentile et al. (2005) have quantified the non-circular motions and found them to be $\sim 2 \mathrm{~km} \mathrm{~s}^{-1}$, i.e., too small by about an order of magnitude to explain the $\Lambda$ CDM discrepancy. An analysis of non-circular motions in 19 THINGS galaxies (Trachternach et al. 2008) also finds similar results: especially in the low-luminosity galaxies, non-circular motions are too small to explain the observed discrepancies.

A different problem is that in order to isolate the dynamical contribution of the dark matter, the mass distribution of the baryons (gas and stars) needs to be determined first. For the neutral gas this can be directly derived from the integrated Hi map, but for the stars this is less trivial. It requires knowledge of the stellar mass-to-light ratio (hereafter $\Upsilon_{\star}$ ) which depends on several factors that are not well constrained, such as the amount of extinction from dust, the star formation history, and the stellar Initial Mass Function. Moreover, these factors are interdependent, making $\Upsilon_{\star}$ one of the parameters in galaxy mass modeling with the largest uncertainty. Therefore, in many studies, minimum and maximum disk assumptions are often used (van Albada \& Sancisi 1986): the minimum disk assumes that the observed rotation curve is entirely due to dark matter and thus gives a hard upper limit to the dark matter properties in galaxies. The maximum disk hypothesis maximizes the rotation contribution of the stellar disk and thus provides an upper limit on $\Upsilon_{\star}$ or, equivalently, a lower limit on the contribution of dark matter. However, these assumptions, though they provide useful limits, are not able to determine the exact amount of dark matter in galaxies.

In this paper, we investigate the distribution of dark matter in two nearby dwarf galaxies, IC 2574 and NGC 2366, using observations obtained as part of The Hi Nearby Galaxy Survey (THINGS; Walter et al. 2008). Dwarf galaxies are dark matter dominated and the highly resolved THINGS observations provide an opportunity to trace in detail the overall dynamics and constrain the dark matter distribution. The distorted velocity contours in IC 2574 and NGC 2366 indicate the presence of non-circular motions (see Walter et al. 2008; de Blok et al. 2008; Trachternach et al. 2008). A key element of our analysis is a new approach based on a Gaussian fit algorithm to decompose the Hi profiles. This method reduces and quantifies the effects of random non-circular motions or localized distortions due to, for example, Hi shells and thus helps to extract the velocity 
component representing the underlying "undisturbed" kinematics. In this paper, we only focus on the small-scale non-circular motions due to, e.g., star formation events and which are not related to large-scale features such as non-axisymmetric shapes of potentials etc. (see Trachternach et al. 2008 for a more extensive discussion on these large-scale non-circular motions). The adopted distances of IC 2574 and NGC 2366 are $4.0 \mathrm{kpc}$ and $3.4 \mathrm{kpc}$, respectively (Walter et al. 2008).

We also constrain the mass of the stellar component of IC 2574 by using Spitzer Infrared Array Camera (IRAC) $3.6 \mu \mathrm{m}$ data from the Spitzer Infrared Nearby Galaxies Survey (SINGS; Kennicutt et al. 2003). NGC 2366 is not part of the SINGS sample and for this galaxy we retrieved $3.6 \mu \mathrm{m}$ data from the Spitzer archives. We combine these data with population synthesis models derived using the "GALAXEV" package of Bruzual \& Charlot (2003). We compare mass models derived using our best $3.6 \mu \mathrm{m} \Upsilon_{\star}$ values with models derived under different assumptions, such as maximum or minimum disk.

The organization of this paper is as follows: in Section 2, we give a general description of data used in this paper. We derive the Hi rotation curves of IC 2574 and NGC 2366 using a new Gaussian decomposition method in Section 3. We present our determinations of the masses of the stellar components of IC 2574 and NGC 2366 in Section 4. The derived mass models of IC 2574 and NGC 2366 are presented in Section 5 and fitted using a combination of halo models and $\Upsilon_{\star}$ assumptions. In Section 6, we measure the inner slopes of the mass density profiles of IC 2574 and NGC 2366 and the main results of this paper are summarized in Section 7.

\section{Data}

The Hi Nearby Galaxy Survey (THINGS) is one of the largest HI survey programs undertaken with the NRAO 1 Very Large Array (VLA) and comprises observations of 34 nearby galaxies. It has high spatial $\left(\sim 6^{\prime \prime}\right)$ and spectral $\left(\leq 5.2 \mathrm{~km} \mathrm{~s}^{-1}\right)$ resolutions (Walter et al. 2008). In our analysis we use the natural-weighted cubes. In order to preserve the noise characteristics of the data, no residual scaling, primary beam correction or blanking was applied. The channel separation in the two galaxies discussed here, $\delta V_{\text {channel }}$, is $2.6 \mathrm{~km} \mathrm{~s}^{-1}$. To constrain the contribution of the stellar component to the total kinematics we use $3.6 \mu \mathrm{m}$ data from the Spitzer Infrared Nearby Galaxies Survey (SINGS) (Kennicutt et al. 2003) for IC 2574 and from the Spitzer archives for NGC 2366. These can be used as a proxy for the distribution of the stellar population. The resolution of $\sim 4^{\prime \prime}$ of the $3.6 \mu \mathrm{m}$ images is comparable to the THINGS resolution. In addition, we use ancillary optical $B, V$, and $R$ images taken with the $2.1 \mathrm{~m}$ telescope at Kitt Peak National Observatory (KPNO) as part of the SINGS survey. We show the data for IC 2574 and NGC 2366 in Fig. 1.

\footnotetext{
${ }^{1}$ The National Radio Astronomy Observatory is a facility of the National Science Foundation operated under cooperative agreement by Associated Universities, Inc.
} 


\section{Bulk velocity fields}

\subsection{The bulk-motion extraction method}

There are several ways to define a velocity field. These include first-moment maps, single Gaussian, multiple Gaussian, or hermite polynomial fits, as well as peak velocity fields (see de Blok et al. 2008 for a detailed discussion). Some, such as first-moment maps, Gaussian fits, and peak velocity fields, work well for single, symmetrical Hi profiles, but fail to properly take into account the presence of multiple velocity components. In the upper-left panels of Fig. 2 and 3 we show the intensity-weighted mean first-moment (hereafter IWM) velocity fields of IC 2574 and NGC 2366 (the "standard" velocity fields). From the distorted contours in these velocity fields, it is clear that there are strong non-circular motions in both galaxies. It is also clear that the IWM velocity fields are not optimal for deriving a rotation curve and/or correcting for the non-circular motions. One can try and capture these non-circular motions by fitting multiple Gaussian components to the profiles. However, even though it is possible to model a non-Gaussian profile using multiple Gaussian components, the standard method often implemented in astronomical software packages will frequently fail as it tries to fit these components simultaneously. This usually requires too many free parameters (i.e., amplitude, dispersion, and central position for each component used), quickly exceeding the typical number of data points in an Hi profile. In addition, the presence of noise can result in the fit becoming sensitive to the values of the initial estimates. However, even if profiles are decomposed perfectly, we are still left with the fundamental problem that a decomposition into Gaussian components does not give much insight as to which component is more representative of underlying circular rotation of the disk and which one represents non-circular motion or additional velocity components along the line of sight. In order to address this problem and extract the undisturbed "bulk motion" (i.e., the velocity most representative of the undisturbed rotation) from the Hi data cube of a given galaxy, we devised an alternative Gaussian decomposition method. This method minimizes the effects of localized non-circular motions (such as those caused by star formation processes) and extracts the circularly rotating components from the Hi data cube. The method consists of the following steps.

\subsubsection{Step I: Estimate the initial, approximate rotation curves}

As an initial step, an approximate rotation curve is derived from a major axis position-velocity diagram. For this we need to derive the position angle (PA) of the major axis, but also the center coordinates (XPOS, YPOS), inclination (INCL) and systemic velocity (VSYS).

Initial estimates for XPOS, YPOS, PA, and INCL can be derived from ellipse fits to isophotes in

the HI surface density-map ( $0^{\text {th }}$ moment map), optical, or infrared images. This gives approximate but reasonable initial values. In this paper, we used ellipse fits to the $3.6 \mu \mathrm{m}$ images of IC 2574 and NGC 2366 at a level of $0.03 \mathrm{MJy} \mathrm{sr}^{-1}$ to determine these initial values. We show the ellipse fits to 
the $3.6 \mu \mathrm{m}$ images for IC 2574 and NGC 2366 in the middle panels of Fig. 1. An estimate for VSYS is determined by averaging the velocity values found near the center position. Using the obtained geometric parameters, we perform single Gaussian fits to the profiles found along the major axis in the Hi data cube, and extract the rotation velocity (VROT). If multiple peaks with different velocities exist at a given spatial position along the major axis, we select one of them as the initial estimate for the bulk motion, as determined by the average velocity at neighboring positions. We emphasize that at this stage we are only determining a first approximation to the rotation velocity and any errors introduced by an incorrect selection of a velocity component will be corrected in subsequent steps.

\subsubsection{Step II: Create the model velocity field for the bulk motion}

We now create an artificial velocity field using the previously obtained geometrical parameters. As we are at this stage only interested in the overall shape of the rotation curve, we approximate the values of VROT obtained above with a fifth-order polynomial. The model velocity field will be used as initial estimates for extracting the bulk motions. The model fields for IC 2574 and NGC 2366 are shown in the top-right panels of Figs. 2 and 3, respectively. Again, we stress that these model velocity fields are just first approximations. They are merely used to set the initial values for carrying out the multiple Gaussian decomposition described in the following step.

\subsubsection{Step III: Perform the single Gaussian fit}

We now perform single Gaussian fits to extract the typical velocities of all profiles in the data cube, taking into account the presence of possible secondary components. For this, we developed a program written in C. As is illustrated in the middle panel of Fig. 4, if a profile consists of two Gaussian components, the primary (i.e., strongest intensity) Gaussian component is extracted as shown, taking into account the effect of the secondary component on the fit. To do this, the program only uses data points that are least affected by the secondary component (as shown by the filled circles in the middle panel of Fig. 4).

We can judge the effect of the secondary component by making Gaussian fits to both halves of the primary Gaussian component separately (as defined with respect to the position of the peak flux). The fit to the side containing the secondary component will result in a larger dispersion compared to the side with only a single component. This difference can be used to indicate the approximate location of the secondary component in a profile. 


\subsubsection{Step IV: Extract the bulk velocity field via the multiple Gaussian decomposition}

In the last step, we assess whether the extracted velocities of the profiles in Step III are acceptable as representative of the underlying circular rotation of the disk. For this, we compare those velocities with the model velocity fields derived in step II. If at some position the velocity difference $(\Delta V)$ is less than a prescribed limit (here we use $3 \delta V_{\text {channel }}$ ), we select the velocity derived in step III for the primary component as the velocity of the bulk motion at that position.

However, if the difference is larger, this suggests that a better velocity can be found by considering a secondary component, and we proceed as follows. The primary Gaussian component of the profile in step III is subtracted from the original profile and subsequently a single Gaussian fit is made to the residual profile, yielding a second Gaussian component. This is illustrated in the right-most panel of Fig. 4. When performing this second Gaussian fit, the model velocity field created in step II is used to determine an initial fit value. We compare the velocity derived for the secondary component with the model velocity field derived. Likewise, we choose this velocity as the bulk velocity at this position, if the velocity difference $\Delta V$ is less than a certain limit (where we again use $\left.3 \delta V_{\text {channel }}\right)$. In cases where the velocity difference is larger, and no satisfactory primary or secondary component is found, we simply put a blank value in that position. We also note that the extracted fits that satisfy our $\Delta V<3 \delta V_{\text {channel }}$ criterion, are only defined as the "bulk velocity" component if they have a significant flux. We demand a peak flux $>3 \sigma$, where $\sigma$ is the rms noise in the cube. We thus substitute a blank value into the bulk velocity field at positions where the extracted velocity shows strong deviations or where no significant emission can be found. This means that our method does not "invent" data where there are none. Using these procedures we have now extracted the first approximate bulk velocity field from the Hi data cube.

We now repeat the entire process and go back to step I. However, rather than using the major axis estimates, we now fit a full "tilted ring model" (Begeman 1989) to the extracted bulk velocity field obtained in step $I V$ and derive a second approximation to the rotation curve parameters (XPOS, YPOS, VSYS, PA, INCL, and VROT). Using these newly determined tilted ring parameters, we construct a second, improved model velocity field for the bulk motion, which is less affected by additional components along the line of sight, and then proceed to steps II, III, and $I V$. In this way, we iterate the above procedures until the mean difference (after one round of $3 \sigma$ outlier rejection) between successive velocity fields is less than $3 \delta V_{\text {channel }}$. We have tested this method using different initial conditions (model velocity fields) and find that the result is independent of these conditions. Empirically it was determined that three or four iterations lead to a stable result. The procedure is summarized in Fig. 5.

The bulk velocity method works particularly well for galaxies in which non-circular motions

form a significant part of the rotation velocity. For galaxies with high rotation velocities, the method produces results very similar to the hermite polynomial method for constructing velocity fields described in de Blok et al. (2008). 


\subsection{The bulk velocity fields of IC 2574 and NGC 2366}

We applied this method to IC 2574 and NGC 2366. The final extracted bulk velocity fields are shown in the bottom-left panels of Figs. 2 and 3, respectively. Most disturbances seen in the IWM velocity fields of both galaxies are removed, especially in the central regions of IC 2574 and the north-western part of NGC 2366. In general a warp significantly changes the position angle of the kinematical major axis as a function of radius and is found in the outer parts of a galaxy (Bosma 1978). No significant changes of the derived position angles, as will be shown in Section 3.3, of the tilted ring models of IC 2574 and NGC 2366 are shown in regions where most disturbances are seen. Therefore, these disturbances are not likely to be from a warp. As a bonus, we also obtain the velocity fields of the strong non-circular motions, which are shown in the bottom-right panels of Figs. 2 and 3. These contain the velocities of the primary components at the positions where these primary components were found to track the non-circular motions, i.e., this is not a residual velocity field. In order to visualize how well the extracted bulk velocity field traces the rotation of the gas, we use azimuthal position-velocity diagrams measured along ellipses defined using the tilted ring models. In a quiescent galaxy with only circular motions, one expects the observed velocities to follow a cosine as a function of azimuth. Any regions containing non-circular motions deviate from the cosine curves and we can therefore use the position-velocity diagrams to assess whether the bulk-motion extraction method is able to properly decompose the multiple profiles

and identify the bulk motion component properly (see Stil \& Israel 2002 for an analysis which uses these azimuthal position-velocity diagrams to quantify non-circular motions).

In Figs. 6 and 7, we show the azimuthal position-velocity diagrams of IC 2574 and NGC 2366, respectively. In Fig. 6, strong non-circular motions in IC 2574 are easily identified at $\theta \sim 320^{\circ}$ at most radii. For example, in the region at $R \sim 228^{\prime \prime}$ and $\theta \sim 320^{\circ}$, two clearly separated profiles are visible. This is where the IWM velocities are strongly affected by non-circular motions. These noncircular motions are caused by the effects of the supergiant shell in IC 2574 (discussed in Walter et al. 1998). In contrast, the bulk velocities extracted by our method follow a sinusoidal line tracing the underlying bulk motion of IC 2574. Similarly, for NGC 2366 in Fig. 7 we can see the presence of strong non-circular motions at $\theta \sim 270^{\circ}$ at most radii. The IWM velocities, affected by non-circular motions, display a non-symmetric shape in the position-velocity diagram in regions with $R>300^{\prime \prime}$ and $\theta \sim 270^{\circ}$. The extracted bulk velocities again have a sinusoidal-shape, indicating that the bulk velocity field for NGC 2366 indeed traces the underlying rotation.

\subsection{Hi rotation curves}

We derived the rotation curves of IC 2574 and NGC 2366 using the bulk velocity fields obtained in Section 3.2. In order to quantify the difference with the IWM velocity field, we also derive the rotation curves from the latter velocity fields.

We used the GIPSY task ROTCUR to make tilted ring fits to the bulk and IWM velocity fields. 
The initial estimates of the ring parameters, such as XPOS, YPOS, PA, and INCL were determined by an ellipse fit to the IRAC $3.6 \mu \mathrm{m}$ images for IC 2574 and NGC 2366 (except VSYS which was derived from the Hi global profile). After fixing the position of the dynamical center (XPOS, YPOS) and VSYS, we run ROTCUR with PA and INCL as free parameters. We made low-order polynomial fits (usually of fifth order) to the PA and INCL distributions to describe the large-scale variations with radius. We fixed PA and INCL to these fit values and ran ROTCUR again with XPOS, YPOS, and VSYS as free parameters. From this, we radially average the XPOS, YPOS, and VSYS and obtain more fine-tuned mean values. These values were fixed again and ROTCUR was run once more leaving the PA and INCL free. We iterated the ROTCUR task in this way until all ring parameters converged. For the last step, we fixed all ring parameters except VROT and derived the final rotation curves of IC 2574 and NGC 2366. The derived rotation curves are shown in Figs. 8 and 9 and the tilted ring parameters are summarized in Table 1.

In the case of IC 2574 we found that the maximum difference between rotation velocities as derived from the IWM and bulk velocity fields is about $\sim 14 \mathrm{~km} \mathrm{~s}^{-1}$ at a radius of around $7 \mathrm{kpc}$, as shown in Fig. 10 and Fig. 11 and this is a significant difference. Note also the decreased scatter in the tilted ring parameters, compared to the IWM tilted ring model presented in Fig. 10. This can be considered as circumstantial evidence that the bulk velocity field is less affected by non-circular motions and therefore traces the underlying rotation of IC 2574 more accurately than the IWM velocity field.

We note the large difference between the bulk velocity field rotation curve and the rotation curve derived by Martimbeau et al. (1994) in an earlier study (Fig. 11). Martimbeau et al. (1994) used an IWM velocity field and adopted a large value for the inclination $\left(\sim 75^{\circ}\right)$, both of which are responsible for this difference.

We compare the rotation curve of NGC 2366 derived from the bulk velocity field with the one derived from the IWM velocity field in Fig. 12. The derived geometrical tilted ring parameters from both bulk and IWM velocity fields are consistent with each other, except for small differences in the position angle. We do find a significant velocity difference in the outer parts. Larger noncircular motions thus exist in the outer parts of NGC 2366 confirming the impression given by the bottom-right panel of Fig.3.

Fig. 13 compares our bulk rotation curve of NGC 2366 with previous determinations by Hunter et al. (2001) and Swaters (1999). The latter curve agrees with the current data out to $5.5 \mathrm{kpc}$. The Hunter et al. (2001) curve is systematically lower than our bulk curve. A partial explanation is that they used a higher inclination of $65^{\circ}$, but this difference is not large enough to explain the entire discrepancy. Though Hunter et al. (2001) do not explicitly state how their velocity field was constructed, it is likely that they used an IWM map, rather than one based on profile fits.

Declining rotation curves at the outer parts of a galaxy have been often considered as an indication that the end of the dark matter distribution is reached (Carignan \& Puche 1990; Casertano \& van Gorkom 1991; Ryder et al. 1997; Carignan \& Purton 1998). However, as shown in Fig. 13, 
the possibility that a declining rotation curve at the outer parts of a galaxy is simply mimicked by non-circular motions cannot be ruled out.

The comparisons between IWM and bulk velocity results given above have to some degree been qualitative. To really quantify the presence of non-circular motions in both types of velocity fields and gauge the effectiveness of the bulk velocity method in removing these effects, we show here harmonic decompositions of the IWM and bulk velocity fields of IC 2574. We refer to Trachternach et al. (2008) for an extensive discussion of the method. In summary, we used the GIPSY task RESWRI to decompose the velocity fields into sine and cosine terms and only kept the center position fixed during the harmonic expansion. We only included terms up to third order. The line-of-sight velocity then has the following form:

$$
v_{\text {los }}(R)=v_{\text {sys }}+\sum_{n=1}^{3}\left[c_{n}(R) \cos n \psi+s_{n}(R) \sin n \psi\right]+\text { resid. }
$$

where $c_{0}$ represents the systemic velocity, and $c_{1}$ the rotation velocity, the $c_{2}, c_{3}, s_{1}, s_{2}$, and $s_{3}$ components quantify non-circular motions. For us, the total amplitudes of the non-circular motions are of most interest. The median absolute amplitudes $\langle A\rangle$ of each component were calculated by taking the median of $A_{n}(R)$, where

$$
A_{n}(R)=\sqrt{c_{n}(R)^{2}+s_{n}(R)^{2}}
$$

for $n>1$, and

$$
A_{1}(R)=\sqrt{s_{1}(R)^{2}},
$$

for $n=1$ ( $c_{1}$ is the rotation velocity). As can be seen in the top-left panel of Fig. 14, the radially averaged, median amplitudes for the $n=1$, and $n=3$ components are small for both velocity fields. For the second order $(n=2)$, however, the harmonic decomposition of the bulk velocity field results in a much smaller amplitude than that of the IWM velocity field. The effect of the bulk velocity method can be seen even more clearly in the top-center panel of Fig. 14. Here, we plot the absolute amplitude of the non-circular motions using the following equation:

$$
\langle A(R)\rangle=\sqrt{s_{1}(R)^{2}+c_{2}(R)^{2}+s_{2}(R)^{2}+c_{3}(R)^{2}+s_{3}(R)^{2}}
$$

The harmonic components found in the IWM velocity field show large amplitudes between 150 " < $R<350^{\prime \prime}$, whereas the results from the bulk velocity field are consistent with regular circular rotation.

\subsection{The mass model for the gas component}

The rotation curves obtained from the bulk velocity field reflect the total (baryonic + dark matter) kinematics of galaxies and in order to say anything about the dark matter, we thus need to quantify the contribution by the baryons. 
To derive the contribution of the gas component to the total kinematics the radial Hi surface density distribution is required. This is obtained from the integrated HI map (natural-weighted) (Walter et al. 2008) using the derived tilted ring model parameters. The Hi radial surface density is then used to derive the corresponding contribution to the rotation velocity after correcting the surface density for the presence of helium and metals (i.e., after scaling by a factor of 1.4; de Blok et al. 2008). We use the task ROTMOD implemented in GIPSY and assume an infinitely thin disk. The rotation velocities for the gas component of IC 2574 and NGC 2366 are presented in the bottom-left panels of Figs. 19 and 20, respectively.

\section{Stellar component}

\subsection{The $3.6 \mu \mathrm{m}$ surface brightness profile}

After removing bright foreground stars in the vicinity of IC 2574 and NGC 2366 manually, the $3.6 \mu \mathrm{m}$ surface brightness profiles are determined using the ELLINT task in GIPSY and the tilted ring fit parameters derived in Section 3.3. We used the standard IRAC calibration which provides

flux values in units of $\mathrm{MJy} \mathrm{sr}^{-1}$. To convert this to surface brightness units of mag $\operatorname{arcsec}^{-2}$ we use:

$$
\mu_{3.6 \mu \mathrm{m}}=-2.5 \times \log _{10}\left[\frac{S_{3.6 \mu \mathrm{m}} \times 2.35 \times 10^{-5}}{\mathrm{ZP}_{3.6 \mu \mathrm{m}}}\right],
$$

where $S_{3.6 \mu \mathrm{m}}$ is the flux value of the $3.6 \mu \mathrm{m}$ band in units of $\mathrm{MJy} \mathrm{sr}^{-1}$. $\mathrm{ZP}_{3.6 \mu \mathrm{m}}$ is the IRAC zero magnitude flux density in Jy and has 280.9 (Reach et al. 2005). The final surface brightness profiles for IC 2574 and NGC 2366 in the $3.6 \mu \mathrm{m}$ band are shown in the top-left panel of Fig. 15 and Fig. 16 , respectively.

\section{2. $\quad$ Determining the $3.6 \mu \mathrm{m}$ and $4.5 \mu \mathrm{m} \Upsilon_{\star}$ values}

In general, the $\Upsilon_{\star}$ values at optical wavelengths are affected by many factors, including dust, age, metallicity, IMF, and recent star formation. These give rise to large uncertainties in the inferred $\Upsilon_{\star}$ values with corresponding uncertainties in dark matter halo parameters. To circumvent this problem usually some assumptions for the value of $\Upsilon_{\star}$, such as minimum disk or maximum disk are used when performing disk-halo decompositions (van Albada \& Sancisi 1986; see discussion in de Blok et al. 2008). However, the derived dark matter halo properties derived using these minimum/maximum disk assumptions are, as discussed above, only upper/lower limits of the properties on the dark matter halo.

Optical colors of the disk are also often used to put further constraints on $\Upsilon_{\star}$. A relation between optical colors (e.g., $B-R, B-V$ ) and $\Upsilon_{\star}$ has been found in earlier work (e.g., Bell \& de Jong 2001). However these previous studies do not provide the $\Upsilon_{\star}$ values for the IRAC bands, so 
we cannot use them here. We have therefore calculated the $\Upsilon_{\star}$ values in the $3.6 \mu \mathrm{m}$ and $4.5 \mu \mathrm{m}$ bands. For this calculation we constructed stellar population synthesis models, with various sets of metallicity and star formation histories using the "GALAXEV" package of Bruzual \& Charlot (2003). Assuming an age of 12 Gyrs (as found for the Local Group in Whiting 1999), we find a well-defined relation between $\Upsilon_{\star}^{K}$ and $\Upsilon_{\star}$ in the $3.6 \mu \mathrm{m}$ and $4.5 \mu \mathrm{m}$ bands as shown in Fig. 17. These can be parameterized as follows,

$$
\Upsilon_{\star}^{3.6}=B^{3.6} \times \Upsilon_{\star}^{K}+A^{3.6}
$$

for the $3.6 \mu m$ band and,

$$
\Upsilon_{\star}^{4.5}=B^{4.5} \times \Upsilon_{\star}^{K}+A^{4.5}
$$

for the $4.5 \mu \mathrm{m}$ band with coefficients, $A^{3.6}, B^{3.6}, A^{4.5}$, and $B^{4.5}$ as given in Table 2 . Bell \& de Jong (2001; their Table 4) give the relations between the $\Upsilon_{\star}^{K}$ and optical colors and we have:

$$
\log _{10}\left(\Upsilon_{\star}^{K}\right)=b^{K} \times \text { Optical Color }+a^{K},
$$

where $a^{K}$ and $b^{K}$ are also given in Table 2 for the appropriate colors.

Combining Eq. 6 with Eq. 8, adopting 20\% solar metallicity (Miller \& Hodge 1996) and a scaled Salpeter IMF cutting off the stars less massive than $\sim 0.35 M_{\odot}$ (Bell \& de Jong 2001), we calculated $\Upsilon_{\star}$ for IC 2574 in the $3.6 \mu \mathrm{m}$ band. As mentioned in Section 2, we use the ancillary optical $B, V$ and $R$ images to derive the radial color distribution of IC 2574 . In the outer parts of the galaxy, we extrapolate the surface brightness profile using exponential fits. From these fits we extract optical colors $(B-R$ and $B-V)$ of IC 2574 as shown in the top-right panel of Fig. 15 . The colors become bluer with increasing radius, consistent with earlier results found for spiral galaxies (de Jong 1996). In general, these color gradients can be explained by different star formation histories and thus different present-day stellar populations, with relatively older populations in the inner parts than in the outer parts. This population change therefore also implies radial changes in $\Upsilon_{\star}$ in galaxies. In order to reflect this $\Upsilon_{\star}$ variation, we take the color gradient into account when determining $\Upsilon_{\star}$. The resulting trends are shown in the bottom-left panel of Fig. 15 . To determine our final $\Upsilon_{\star}$ values, we simply average the $\Upsilon_{\star}^{K}$ values as derived from $B-R$ and $B-V$, respectively. The average $\Upsilon_{\star}^{3.6}$ used for the final mass model of the stellar component of IC 2574 is shown in the bottom-right panel of Fig. 15 (gray solid line).

Similarly, we calculated $\Upsilon_{\star}$ values in the $3.6 \mu \mathrm{m}$ band for NGC 2366 using Eqs. 6 and 8. For NGC 2366, we used a 10\% solar metallicity (Hunter et al. 2006) and a scaled Salpeter IMF. We used the optical color $(B-V)$ of NGC 2366 given in Hunter et al. (2006) and found the coefficients of $a^{K}$ and $b^{K}$ as shown in Table 2. We used a constant average color, $(B-V)=0.31$, since the radial $B-V$ color distribution given in Hunter et al. (2006) is nearly constant (except where the supergiant Hil region of NGC 2363 is located at a radius $\sim 1 \mathrm{kpc}$ ). From this we obtained $\Upsilon_{\star}^{3.6}=0.33$ for NGC 2366 as given in Table 2. See also de Blok et al. (2008) for a comparison between the stellar disk masses of a number of THINGS galaxies derived using our method and the approach adopted by Leroy et al. (2008). 
The $3.6 \mu \mathrm{m}$ emission may contain contributions from PAH features but, in the case of the dwarfs, the contribution of PAHs is not likely to be very high (see Walter et al. 2007 for a study of the SINGS dwarfs). The $3.6 \mu \mathrm{m}$ emission is likely to be a good proxy for the old stellar population even if there can be contributions from the intermediate-age population like AGB stars. Although the $3.6 \mu \mathrm{m}$ images thus provide a virtually dust-free picture of the stellar component of a galaxy, the optical colors that are implicitly used to determine $\Upsilon_{\star}^{3.6}$ are possibly affected by dust. However, Bell \& de Jong (2001) show that $\Upsilon_{\star}^{K}$ is only weakly dependent on optical color, in contrast with $\Upsilon_{\star}^{B}$. This is illustrated by the low values of $b^{K}$ in the IC $2574 \Upsilon_{\star}^{K}$ relation $(\sim 0.6$ for $B-V$ and $\sim 0.4$ for $B-R$ ), especially when compared with the much higher $b^{B}$ values listed in Bell \& de Jong (2001). The precise value of $\Upsilon_{\star}^{3.6}$ is thus not very sensitive to variations in $B-V$ or $B-R$.

\subsection{The mass model for the stellar components}

The $3.6 \mu \mathrm{m}$ surface brightness profiles derived in Section 4.1 are in units of $\mathrm{mag} \operatorname{arcsec}^{-2}$, and must, using the values of $\Upsilon_{\star}$ derived in Section 4.2 , still be converted to a mass density profile in units of $M_{\odot} \mathrm{pc}^{-2}$. The conversion to mass surface density is calculated with the following formula:

$$
\Sigma\left[M_{\odot} \mathrm{pc}^{-2}\right]=\Upsilon_{\star}^{3.6} \times 10^{-0.4 \times\left(\mu_{3.6 \mu \mathrm{m}}-C^{3.6}\right)},
$$

where $C^{3.6}$ is a constant for converting mag $\operatorname{arcsec}^{-2}$ to $L_{\odot} \mathrm{pc}^{-2}$. We have:

$$
\begin{aligned}
-2.5 \log _{10}\left(1.0 L_{\odot} \mathrm{pc}^{-2}\right) & \simeq-2.5 \log _{10}\left(1.0 L_{\odot}\right)+21.56 \\
& =M_{\odot}^{3.6}+21.56 \\
& =C^{3.6}
\end{aligned}
$$

where $M_{\odot}^{3.6}$ is the absolute solar magnitude in the $3.6 \mu \mathrm{m}$ band. To calculate this, we perform the following steps.

\section{The apparent magnitude of the Sun}

The apparent magnitude of the Sun, $m_{\odot}^{\lambda}$, in the $3.6 \mu \mathrm{m}$ or $4.5 \mu \mathrm{m}$ band is given by:

$$
m_{\odot}^{\lambda}=-2.5 \log _{10}\left[\int_{0}^{\infty} \mathrm{d} \lambda R(\lambda) f_{\odot}^{\lambda}\right]+2.5 \log _{10}\left[\int_{0}^{\infty} \mathrm{d} \lambda R(\lambda) f_{\alpha \mathrm{Lyr}}^{\lambda}\right],
$$

where $R(\lambda)$ is the filter response function and $f_{\odot}^{\lambda}$ and $f_{\alpha \text { Lyr }}^{\lambda}$ are the spectral energy distributions of the Sun and $\alpha$ Lyr respectively (Fukugita et al. 1995). The Kurucz model (1992) for $\alpha$ Lyr has $T_{\text {eff }}=9400 \mathrm{~K}, \log _{10} g=3.90$, and $\log _{10} Z=-0.50$. The convolved spectral energy distributions of the Sun and $\alpha$ Lyr using the IRAC filter response functions in the $3.6 \mu \mathrm{m}$ and $4.5 \mu \mathrm{m}$ bands are shown in Fig. 18. We compute the areas below these convolved spectral energy distributions of the Sun and $\alpha$ Lyr. Using Eq. 11, we then obtain the apparent magnitude of the Sun as -28.33 and -28.30 
in the $3.6 \mu \mathrm{m}$ and $4.5 \mu \mathrm{m}$ bands, respectively.

\section{The absolute magnitude of the Sun in the IRAC bands}

We obtain the absolute magnitude of the Sun as follows,

$$
m_{\odot}^{\lambda}-M_{\odot}^{\lambda}=5 \log _{10} D_{\odot}-5,
$$

where $D_{\odot}=1 / 206625$ pc (distance to the Sun). From this, we find

$$
\begin{aligned}
& M_{\odot}^{3.6}=m_{\odot}^{3.6}+31.57=3.24 \\
& M_{\odot}^{4.5}=m_{\odot}^{4.5}+31.57=3.27
\end{aligned}
$$

From Eq. $10, C^{3.6}=24.80$, and using Eq. 9 we then convert the $3.6 \mu \mathrm{m}$ surface brightness to a mass density in units of $M_{\odot} \mathrm{pc}^{-2}$. The final mass models for IC 2574 and NGC 2366 using the mass density for the stellar component are constructed with the ROTMOD task in GIPSY. These are illustrated in Fig. 19 and Fig. 20. For the stellar disk, we assume a vertical sech $^{2}$ scale-height distribution with $h / z_{0}=5$ (van der Kruit \& Searle 1981) where $h$ is the radial scale-length and $z_{0}$ is the scale-height of disk in kpc. This $h / z_{0}=5$ ratio as determined in van der Kruit \& Searle (1981) is based on a small number of galaxies but has, for a much larger sample, been confirmed by Kregel et al. (2002), who find $h / z_{0}=4.8 \pm 1.3$. From exponential disk fits to the $3.6 \mu \mathrm{m}$ surface brightness distributions (top-left panel of Fig. 15 and Fig. 16) we obtain scale-height values of IC 2574 and NGC 2366 of $z_{0}=0.57$ and $0.34 \mathrm{kpc}$, respectively.

\section{Dark matter distribution}

\subsection{Dark matter halo models}

The properties of the dark matter halo of a galaxy are usually quantified by using dark matter halo models. The residuals obtained by subtracting from the derived rotation curves those contributions corresponding to the stellar and gas components, are assumed to be due to the dark

matter halo. In this paper, we explore two models: the $\Lambda$ CDM NFW cusp-dominated halo and the pseudo-isothermal core-dominated halo. Their properties are given below.

\subsubsection{NFW dark matter halo model}

Navarro, Frenk \& White (1996, 1997; NFW) give a prescription for the dark matter distribution found in numerical simulations, based on the CDM paradigm. This so-called "universal density profile" has a cusp feature towards the galaxy center. The profile has the form

$$
\rho_{\mathrm{NFW}}(R)=\frac{\rho_{i}}{\left(R / R_{s}\right)\left(1+R / R_{s}\right)^{2}},
$$


where $\rho_{i}$ is the initial density of the universe at the time of collapse of the halo and $R_{s}$ is the characteristic radius of the dark matter halo. The rotation velocity corresponding to the NFW halo density is given as

$$
V_{\mathrm{NFW}}(R)=V_{200} \sqrt{\frac{\ln (1+\mathrm{cx})-\mathrm{cx} /(1+\mathrm{cx})}{x[\ln (1+\mathrm{c})-\mathrm{c} /(1+\mathrm{c})]}},
$$

where $c$ is the concentration parameter and defined as $R_{200} / R_{s} . V_{200}$ is the rotation velocity at radius $R_{200}$ where the density contrast exceeds 200 and $x$ is defined as $R / R_{200}$. This universal density profile can be approximated using two power laws: $\rho \propto R^{-1}$ at small radii and $\rho \propto R^{-3}$ at large radii. See de Blok et al. (2008) for a more extensive description.

\subsubsection{Pseudo isothermal dark matter halo model}

The spherical pseudo-isothermal halo model which is used in most of the early rotation curve studies is observationally motivated and has a core-like constant density profile which can be described as $\rho \propto R^{0}$ towards the galaxy center and $R^{-2}$ for large $R$. It has the following form,

$$
\rho_{\mathrm{ISO}}(R)=\frac{\rho_{0}}{1+\left(R / R_{c}\right)^{2}},
$$

where $\rho_{0}$ and $R_{c}$ are the core-density and core-radius of the dark matter halo, respectively. This density profile gives the rotation velocity,

$$
V_{\mathrm{ISO}}(R)=\sqrt{4 \pi G \rho_{0} R_{c}^{2}\left[1-\frac{R_{c}}{R} \operatorname{atan}\left(\frac{R}{R_{c}}\right)\right]} .
$$

This approaches the asymptotic velocity at large radii given by

$$
V_{\infty}=\sqrt{4 \pi G \rho_{0} R_{c}^{2}}
$$

\subsection{Mass modeling results}

We now construct mass models using the rotation curves derived from the bulk velocity fields of IC 2574 and NGC 2366 taking into account the distribution of baryons, and use these to fit NFW and pseudo-isothermal halo models. Given the proximity of IC 2574 and NGC 2366, the

THINGS (natural-weighted) resolution of $\sim 12^{\prime \prime}$ corresponds to $\sim 200 \mathrm{pc}$, comparable to that of the simulations of dwarf dark matter halos presented in Navarro et al. (2004). As discussed earlier, differences between NFW and pseudo-isothermal dark matter models are the most distinct in the inner parts of galaxies. Also, as the tilted rings in the outer parts of our galaxies are only partly filled with emission, we estimate the effects of these outer asymmetric features by presenting fits 
to both the entire radial ranges of the rotation curves, as well as fits to the inner parts only (i.e., $R \leq 7.5 \mathrm{kpc}$ for IC 2574 and $R \leq 6 \mathrm{kpc}$ for NGC 2366).

When performing the fits we also explore classical $\Upsilon_{\star}$ assumptions, such as maximum disk, minimum disk, minimum disk + gas (the stellar component is ignored, but the contribution of the gas is taken into account), in addition to the model $\Upsilon_{\star}^{3.6}$ values we calculated. We also attempted a fit with $\Upsilon_{\star}^{3.6}$ as a free parameter. The results are given in Figs. 21 and 22 , and Tables 3 and 4 . The best-fitting unconstrained values for $\Upsilon_{\star}$ differ somewhat from those predicted by the population synthesis modeling described earlier (except for the IC 2574 pseudo-isothermal fit). The negative values of $\Upsilon_{\star}$ that the curves seem to prefer are obviously unphysical. An extensive discussion of this behavior is given in de Blok et al. (2008).

We find that the pseudo-isothermal halo provides a better fit than the NFW halo. NFW models resulted in inferior fits, independent of which value for $\Upsilon_{\star}$ was adopted. The preferred $c$ values (concentration parameter) are all negative with extremely large uncertainties. When making the final fits, we fixed these values to be $c=0.1$. See de Blok et al. (2008) for a discussion on the $c$-values found within the THINGS sample. In contrast, the pseudo-isothermal halo models provide reasonable halo parameters, except for the IC 2574 maximum disk case. However, we found that the fit is very sensitive to the choice of $\Upsilon_{\star}^{\max }$ for the maximum disk and sensible parameters can be obtained by slightly lowering $\Upsilon_{\star}^{\max }$. These results show that the NFW distribution is not an appropriate fitting function for our galaxies and that the implied dark matter distributions of the two dwarf galaxies, IC 2574 and NGC 2366, instead show a sizeable central constant-density core, which can be well approximated by the pseudo-isothermal halo model.

We now compare the Hi rotation curves and mass modeling based on the bulk velocity fields with a similar analysis done using the IWM velocity fields to examine the effect of non-circular motions on the dynamics of galaxies. First, for convenience, we introduce some notation. We use $V_{\text {bulk }}$ to indicate the rotation curve derived from tilted ring fits using the bulk velocity field, as described in Section 3.3. We use $V_{\text {IWM }}^{\prime}$ to describe the rotation curve derived assuming the geometrical parameters from the bulk velocity field tilted ring model but applied to the IWM velocity field. Finally, we use $V_{\text {IWM }}$ to describe the rotation curve derived using a tilted ring model as derived from and applied to the IWM velocity field.

For a galaxy affected by non-circular motions, the traditional IWM velocity field will be distorted. In general, non-circular motions as present in galaxies, are likely to make the observed rotation velocity fall below the circular velocity (Rhee et al. 2004). This is indeed happening in our galaxies as indicated in Fig. 23, where $V_{\text {IWM }}$ is significantly lower than $V_{\text {bulk }}$. The velocity difference between $V_{\text {bulk }}$ and $V_{\text {IWM }}^{\prime}$ can only be attributed to differences in the bulk and IWM velocity fields, as we use otherwise identical geometrical parameters.

In the case of IC 2574, a distinct velocity difference is seen in the central region where the IWM velocity field is distorted by non-circular motions (Fig. 23, left column). Such a velocity difference is also found in NGC 2366, particularly in the outer parts (see the Fig. 23, right column). 
In practice, this underestimate of the rotation velocity results in a decreased dynamical contribution of the dark matter to the total dynamics, as the contribution of stellar and gas components are fixed for any given $\Upsilon_{\star}$ assumption. Consequently, non-circular motions decrease the role of dark matter with respect to the visible matter. In the bottom-left panel of Fig. 23 the rotation velocity due to the stellar component using the model $\Upsilon_{\star}^{3.6}$ values for IC 2574 already exceeds $V_{\text {IWM }}^{\prime}$ and $V_{\text {IWM }}$ in the inner parts even without the dynamical contribution of the gas component. The $V_{\text {bulk }}$ rotation curve, however, can in the inner parts not only accommodate the rotation of the stellar component, but also that of the gas component.

\section{Dark matter mass density profiles}

An intuitive way to illustrate the dark matter distribution of a galaxy is to calculate the mass density that corresponds to the observed rotation velocity. The observed rotation velocity is converted to mass density by assuming a spherical mass distribution (i.e., $\nabla^{2} \Phi=4 \pi G \rho, \Phi=$ $-G M / R)$. For the inversion, we use the following formula (de Blok et al. 2001),

$$
4 \pi G \rho(R)=2 \frac{V}{R} \frac{\partial V}{\partial R}+\left(\frac{V}{R}\right)^{2},
$$

where $V$ is the rotation velocity observed at radius $R$. This direct conversion is only valid under the assumption that the observed rotation velocity is entirely due to the dark matter component, as is the case with a minimum disk (i.e., maximum halo). In general the minimum disk assumption is a good description for dwarf galaxies and LSB galaxies (de Blok et al. 2002), and we therefore also apply this assumption to IC 2574 and NGC 2366. While one could, in principle, make an explicit correction for the rotational contribution of the baryons, for the crucial innermost parts this involves using the difference between two small numbers, which can lead to wildly fluctuating values of the derivative in Eq. 20. The minimum disk assumption thus yields a safe and robust upper limit on the dark matter properties and in particular to the steepness of the inner slope (de Blok \& McGaugh 1997; de Blok et al. 2001).

We follow the method described in de Blok et al. (2002) to determine the slope of the inner component of the mass density profile. We measure the slopes of the inner parts $(R<1.2 \mathrm{kpc})$ of IC 2574 and NGC 2366 using a least squares fit and find the values of the slopes to be $\alpha=+0.13 \pm 0.07$ for IC 2574 and $\alpha=-0.32 \pm 0.10$ for NGC 2366, respectively. These are shown in Fig. 24 and are in good agreement with the earlier result of $\alpha=-0.2 \pm 0.2$ (de Blok et al. 2001, de Blok \& Bosma 2002) for a larger sample of LSB galaxies. These flat slopes thus imply that the dark matter distributions of IC 2574 and NGC 2366 are well characterized by a sizeable constant-density core. The mass density profiles for the best-fit minimum disk NFW and pseudo-isothermal models are also over-plotted in Fig. 24. The pseudo-isothermal halo follows the observed mass density profile most closely. This is in sharp contrast with the steep slope predicted by the NFW profile.

We plot the value of the inner-slope $\alpha$ of the mass-density profile against the observed radius 
of the innermost point $R_{\text {inner }}$ in Fig. 25. The result is consistent with the earlier results by de Blok et al. (2002). In conclusion, the observed dark matter distributions of IC 2574 and NGC 2366 are both best described by the pseudo-isothermal halo model with a constant-density core.

\section{Summary}

We have presented mass models for the nearby dwarf galaxies, IC 2574 and NGC 2366, derived using the high-resolution data from THINGS. These high-resolution data do not suffer from beam smearing, have a well-defined dynamical center and enable us to examine in detail the dark matter distribution of these galaxies. To minimize the effects of random non-circular motions on the derived kinematics of a galaxy, we developed a new Gaussian decomposition method and used this to construct a "bulk" velocity field of IC 2574 and NGC 2366, showing the underlying undisturbed rotation.

The random, non-circular motions of IC 2574 and NGC 2366, visible as distortions in the velocity contours of the traditional IWM velocity fields, were largely removed in the newly constructed bulk velocity fields. Comparing the Hi rotation curves derived from the bulk and IWM velocity fields, we find that the rotation velocities derived from the IWM velocity fields are significantly lower than those from the bulk velocity fields. In addition, non-circular motions of NGC 2366 are likely to be responsible for the declining rotation velocities derived from the IWM velocity fields in the outer parts.

Combining optical and SINGS $3.6 \mu \mathrm{m}$ data, we quantify the dynamical contribution of the stellar component to the total kinematics. For this we compute $\Upsilon_{\star}$ values based on the Bruzual and Charlot (2003) population synthesis models for the $3.6 \mu \mathrm{m}$ and $4.5 \mu \mathrm{m}$ bands.

We have fitted NFW and pseudo-isothermal dark matter halos to the derived rotation curves, taking into account the contributions due to stars and gas. We found that the pseudo-isothermal halo provides a better fit to the observations than the NFW halo. We use the derived mass density profile to determine the value of the inner slope. The measured slopes are $\alpha=+0.13 \pm 0.07$ for IC 2574 and $\alpha=-0.32 \pm 0.10$ for NGC 2366, compared to the NFW model which predicts $\alpha \sim-1$.

The dark matter distributions of IC 2574 and NGC 2366 are well described by the pseudoisothermal model $(\alpha \sim 0)$ with a sizeable central constant-density core. These results are not affected by systematic effects due to lack of resolution or pointing offsets, take into account the effects of non-circular motions and use a well-constrained model for $\Upsilon_{\star}$.

The work of WJGdB is based upon research supported by the South African Research Chairs Initiative of the Department of Science and Technology and National Research Foundation. EB gratefully acknowledges financial support through an EU Marie Curie International Reintegration Grant (Contract No. MIRG-CT-6-2005-013556). This research has made use of the NASA/IPAC 
Extragalactic Database (NED) which is operated by the Jet Propulsion Laboratory, California Institute of Technology, under contract with the National Aeronautics and Space Administration. This publication makes use of data products from the Two Micron All Sky Survey, which is a joint project of the University of Massachusetts and the Infrared Processing and Analysis Center/California Institute of Technology, funded by the National Aeronautics and Space Administration and the National Science Foundation.

\section{REFERENCES}

Begeman, K. 1989, A\&A, 223, 47

Bell, E. F., \& de Jong, R. S. 2001, ApJ, 550, 212

Bosma, A. 1978, PhD Thesis, University of Groningen

Brinks, E., \& Walter, F. 2003, Ap\&SS, 284, 2

Bruzual, G. 1983, ApJ, 273, 105

Bruzual, G., \& Charlot, S. 2003, MNRAS, 344, 1000

Carignan, C., \& Puche, D. 1990, AJ, 100, 394

Carignan, C., \& Purton, C. 1998, ApJ, 506, 125

Casertano, S., \& van Gorkom, J. H. 1991, AJ, 101, 1231

de Blok, W. J. G., \& McGaugh, S. S. 1997, MNRAS, 290, 533

de Blok, W. J. G., McGaugh, S. S., Bosma, A., \& Rubin, V. C. 2001, ApJ, 552, 23

de Blok, W. J. G., \& Bosma, A. 2002, A\&A, 385, 816

de Blok, W. J. G., Bosma, A., \& McGaugh, S. S. 2003, MNRAS, 340, 657

de Blok, W. J. G. 2004, Dark Matter in Galaxies, IAU Symposium 220, 69

de Blok, W. J. G., Walter, F., Brinks, E., Trachternach, C., Oh, S-H., \& Kennicutt, R. C. 2008, AJ, submitted

de Jong, R. S. 1996, A\&A, 313, 377

Flores, R. A., Primack, J. R. 1994, ApJ, 427, L1

Fukugita, M., Shimasaku, K., \& Ichikawa, T. 1995, PASP, 107, 945

Gentile, G., Salucci, P., Klein, U., Vergani, D., \& Kalberla, P. 2004, MNRAS, 351, 903 
Gentile, G., Burkert, A., Salucci, P., Klein, U., \& Walter, F. 2005, AJ, 634, L145

Gentile, G., Tonini, C., Salucci, P. 2007, A\&A, 467, 925

Hayashi, E., \& Navarro, J. F. 2006, MNRAS, 373, 1117

Hunter, D.A., Elmegreen, B.G., van Woerden, H. 2001, ApJ, 556, 773

Hunter, D.A., Elmegreen, B. G. 2006, ApJS, 162, 49

Kennicutt, R. C. et al. 2003, PASP, 115, 928

Klypin, A., Kravtsov, A. V., Valenzuela, O., Prada, F. 1999, ApJ, 522, 82

Kregel, M., van der Kruit, P. C., de Grijs, R. 2002, MNRAS, 334, 646

Kurucz, R. L. 1992, The Stellar Populations of Galaxies, IAU Symposium 149, 225

Kuzio de Naray, R., McGaugh, S. S., de Blok, W. J. G., Bosma, A. 2006, ApJS, 165, 461

Leroy, A. et al. 2008, AJ, submitted

Martimbeau, N., Carignan, C., \& Roy, J.-R. 1994, AJ, 107, 543

McGaugh, S. S., Rubin, V. C., \& de Blok, W. J. G. 2001, AJ, 122, 2381

Miller, B. W. \& Hodge, P. 1996, ApJ, 458, 467

Moore, B. 1994, Nature, 370, 620

Moore, B, Ghigna, S., Governato, F., Lake, G., Quinn, T., Stadel, J., Tozzi, P. 1999, ApJ, 524, 19

Moore, B., Governato, F., Quinn, T., Stadel, J., \& Lake, G. 1998, ApJ, 499, 5

Navarro, J. F., Frenk, C. S., \& White, S. D. M. 1995, MNRAS, 275, 56

Navarro, J. F., Frenk, C. S., \& White, S. D. M. 1996, ApJ, 462, 563

Navarro, J. F., Frenk, C. S., \& White, S. D. M. 1997, ApJ, 490, 493

Navarro, J. F., Hayashi, E., Power, C., Jenkins, A. R., Frenk, C. S., White, S. D. M., Springel, V., Stadel, J., \& Quinn, T. R. 2004, MNRAS, 349, 1039

Primack, J. R. 2003, Nuclear Physics B Proceedings Supplements, 124, 3

Reach, William T., et al. 2005, PASP, 117, 978

Rhee, G., Valenzuela, O., Klypin, A., Holtzman, J., \& Moorthy, B. 2004, ApJ, 617, 1059

Ryder, S. D., Zasov, A., McIntyre, V., \& Walsh, W. 1997, PASA, 14, 85 
Salpeter, E. E. 1955, ApJ, 123, 666

Simon, J. D., Bolatto, A. D., Leroy, A., \& Blitz, L. 2003, ApJ, 596, 957

Spergel, D. N., et al. 2003, ApJS, 148, 175

Stil, J. M., \& Israel, F. P. 2002, A\&A, 392, 473

Swaters, R. A., 1999, PhD Thesis, University of Groningen

Swaters, R. A., Madore, B. F., van den Bosch, F. C., \& Balcells, M. 2003, ApJ, 583, 732

Trachternach, C., de Blok, W. J. G., Brinks, E., Walter, F., \& Kennicutt, R. C. 2008, AJ, submitted van Albada, T. S., \& Sancisi, R. 1986, Philos. Trans. R. Soc. London A, 320, 447

van den Bosch, F. C., Robertson, B. E., Dalcanton, J. J., \& de Blok, W. J. G. 2000, AJ, 119, 1579

van der Kruit, P. C., \& Searle, L. 1981, A\&A, 95, 105

Walter, F., Kerp, J., Duric, N., Brinks, E., \& Klein, U. 1998, ApJ, 502, 143

Walter, F., Brinks, E., de Blok, W. J. G., Bigiel, F., Thornley, M., \& Kennicutt, R. C. 2005, AAS, 207, 6409

Walter, F. et al. 2007, ApJ, 661, 102

Walter, F., Brinks, E., de Blok, W. J. G., Bigiel, F., Kennicutt, R. C., \& Thornley, M. 2008, AJ, submitted

Weldrake, D. T. F., de Blok, W. J. G., \& Walter, F. 2003, MNRAS, 340, 12

Whiting, A. B. 1999, The Stellar Content of Local Group Galaxies, IAU Symposium 192, 420 
Table 1. Parameters of tilted ring models of IC 2574 and NGC 2366

\begin{tabular}{|c|c|c|c|c|c|c|}
\hline Name & $\begin{array}{c}\alpha(2000.0) \\
(\text { hh:mm:ss.s) } \\
(1)\end{array}$ & $\begin{array}{c}\delta(2000.0) \\
(\mathrm{dd}: \mathrm{mm}: \mathrm{ss} . \mathrm{s}) \\
(2)\end{array}$ & $\begin{array}{c}V_{\text {sys }} \\
\left(\mathrm{km} \mathrm{s}^{-1}\right) \\
(3)\end{array}$ & $\begin{array}{l}\langle i\rangle \\
\left({ }^{\circ}\right) \\
(4)\end{array}$ & $\begin{array}{c}\langle\mathrm{PA}\rangle \\
\left({ }^{\circ}\right) \\
(5)\end{array}$ & $\begin{array}{c}D \\
(\mathrm{Mpc}) \\
(6)\end{array}$ \\
\hline NGC 2366 & $07: 28: 53.4$ & $+69: 12: 51.1$ & 104.0 & 63.8 & 39.8 & 3.4 \\
\hline IC 2574 & $10: 28: 27.7$ & $+68: 24: 59.4$ & 53.1 & 53.4 & 55.7 & 4.0 \\
\hline
\end{tabular}

Note. - (1)(2): Center positions. (3): Systemic velocity derived from a tilted ring fit using the bulk velocity field as described in Section 3.3. (4): Average inclination of the tilted ring model. (5): Average position angle of the tilted ring model. (6): Distances as given in Walter et al. (2008). 
Table 2. Coefficients for the $\Upsilon_{\star}$ relations Eqs. 6-8

\begin{tabular}{|c|c|c|c|c|c|c|}
\hline$\lambda$ & \multicolumn{3}{|c|}{$\begin{array}{l}A^{\lambda} \\
(1)\end{array}$} & \multicolumn{3}{|c|}{$\begin{array}{l}B^{\lambda} \\
(2)\end{array}$} \\
\hline $3.6 \mu \mathrm{m}$ & \multicolumn{3}{|c|}{-0.05} & \multicolumn{3}{|c|}{0.92} \\
\hline $4.5 \mu \mathrm{m}$ & \multicolumn{3}{|c|}{-0.08} & \multicolumn{3}{|c|}{0.91} \\
\hline \multirow[b]{2}{*}{ Color } & \multicolumn{3}{|c|}{ IC 2574} & \multicolumn{3}{|c|}{ NGC 2366} \\
\hline & $\begin{array}{l}a^{K} \\
(3)\end{array}$ & $\begin{array}{l}b^{K} \\
(4)\end{array}$ & $\begin{array}{c}\left\langle\Upsilon_{\star}^{3.6}\right\rangle \\
(5)\end{array}$ & $\begin{array}{l}a^{K} \\
(6)\end{array}$ & $\begin{array}{l}b^{K} \\
(7)\end{array}$ & $\begin{array}{c}\left\langle\Upsilon_{\star}^{3.6}\right\rangle \\
(8)\end{array}$ \\
\hline$B-V$ & -0.59 & 0.60 & 0.44 & -0.60 & 0.72 & 0.33 \\
\hline$B-R$ & -0.67 & 0.42 & & $\ldots$ & $\ldots$ & \\
\hline
\end{tabular}

Note. - (1)(2): Coefficients for the relations between $\Upsilon_{\star}^{K}$ and $\Upsilon_{\star}$ in the $3.6 \mu \mathrm{m}$ and $4.5 \mu \mathrm{m}$ bands derived in Section 4.2. (3)(4): Coefficients for the relations between $\Upsilon_{\star}^{K}$ and optical colors given in Bell \& de Jong (2001), adopting 20\% solar metallicity and a scaled Salpeter IMF for IC 2574. (5)(8): Average $\Upsilon_{\star}$ in the $3.6 \mu \mathrm{m}$ band $\left(M_{\odot} / L_{\odot}\right)$. (6)(7): Coefficients for the relation between $\Upsilon_{\star}^{K}$ and optical color given in Bell \& de Jong (2001), adopting 10\% solar metallicity and a scaled Salpeter IMF for NGC 2366. 
Table 3: Parameters of dark halo models for IC 2574

\begin{tabular}{|c|c|c|c|c|c|c|c|c|}
\hline \multirow[b]{2}{*}{$\Upsilon_{\star}$ assumption } & \multicolumn{4}{|c|}{ NFW halo (entire region) } & \multicolumn{4}{|c|}{ NFW halo $(<7.5 \mathrm{kpc})$} \\
\hline & $\begin{array}{c}\left\langle\Upsilon_{\star}^{3.6}\right\rangle \\
(2)\end{array}$ & $\begin{array}{c}c \\
(3)\end{array}$ & $\begin{array}{l}V_{200} \\
(4)\end{array}$ & $\begin{array}{c}\chi_{r e d}^{2} . \\
(5)\end{array}$ & $\begin{array}{c}\left\langle\Upsilon_{\star}^{3.6}\right\rangle \\
(6)\end{array}$ & $\begin{array}{c}c \\
(7)\end{array}$ & $\begin{array}{c}V_{200} \\
(8)\end{array}$ & $\begin{array}{c}\chi_{r e d}^{2} . \\
(9)\end{array}$ \\
\hline Min. disk & 0.00 & $<0.1$ & $674.6 \pm 18.3$ & 2.88 & 0.00 & $<0.1$ & $1213.6 \pm \ldots$ & 3.39 \\
\hline Min. disk+gas & 0.00 & $<0.1$ & $524.3 \pm 51.7$ & 1.65 & 0.00 & $<0.1$ & $1005.5 \pm \ldots$ & 2.32 \\
\hline Max. disk & 0.93 & $<0.1$ & $634.4 \pm \ldots$ & 2.33 & 0.93 & $<0.1$ & $353.8 \pm \ldots$ & 1.63 \\
\hline Model $\Upsilon_{*}^{3.6}$ disk & 0.44 & $<0.1$ & $873.9 \pm \ldots$ & 1.81 & 0.44 & $<0.1$ & $700.5 \pm \ldots$ & 1.96 \\
\hline \multirow[t]{2}{*}{$\Upsilon_{\star}^{\text {free disk }}$} & -0.03 & $<0.1$ & $1107.7 \pm \ldots$ & 1.66 & & & & \\
\hline & \multicolumn{4}{|c|}{ Pseudo-isothermal halo (entire region) } & \multicolumn{4}{|c|}{ Pseudo-isothermal halo $(<7.5 \mathrm{kpc})$} \\
\hline$\Upsilon_{\star}$ assumption & $\begin{array}{c}\left\langle\Upsilon_{\star}^{3.6}\right\rangle \\
(11)\end{array}$ & $\begin{array}{l}R_{C} \\
(12)\end{array}$ & $\begin{array}{c}\rho_{0} \\
(13)\end{array}$ & $\begin{array}{c}\chi_{r e d}^{2} \\
(14)\end{array}$ & $\begin{array}{c}\left\langle\Upsilon_{\star}^{3.6}\right\rangle \\
(15)\end{array}$ & $\begin{array}{c}R_{C} \\
(16)\end{array}$ & $\begin{array}{c}\rho_{0} \\
(17)\end{array}$ & $\begin{array}{c}\chi_{r e d}^{2} \\
(18)\end{array}$ \\
\hline Min. disk & 0.00 & $5.77 \pm 0.16$ & $7.8 \pm 0.2$ & 0.25 & 0.00 & $5.69 \pm 0.35$ & $7.8 \pm 0.3$ & 0.26 \\
\hline Min. disk+gas & 0.00 & $4.61 \pm 0.12$ & $7.6 \pm 0.2$ & 0.16 & 0.00 & $3.88 \pm 0.16$ & $8.7 \pm 0.3$ & 0.13 \\
\hline Max. disk & 0.93 & $27.52 \pm 10.22$ & $1.8 \pm 0.1$ & 0.30 & 0.93 & $\ldots$ & $1.7 \pm 0.9$ & 0.39 \\
\hline Model $\Upsilon_{*}^{3.6}$ disk & 0.44 & $7.23 \pm 0.30$ & $4.1 \pm 0.1$ & 0.17 & 0.44 & $5.87 \pm 0.55$ & $4.5 \pm 0.3$ & 0.19 \\
\hline$\Upsilon_{\star}^{\text {free }}$ disk & 0.10 & $4.99 \pm 0.34$ & $6.7 \pm 0.7$ & 0.16 & & & & \\
\hline
\end{tabular}

Note.-(1)(10): The stellar mass-to-light ratio $\Upsilon_{\star}$ assumptions. "Model $\Upsilon_{\star}^{3.6}$ disk" uses the values derived from the population synthesis models in Section 4.2. $\Upsilon_{\star}^{\text {free }}$ has $\Upsilon_{\star}^{3.6}$ as a free parameter. (2)(6)(11)(15): Average $\Upsilon_{\star}$ in the $3.6 \mu \mathrm{m}$ band $\left(M_{\odot} / L_{\odot}\right)$. (3)(7): Concentration parameter c of NFW halo model (NFW 1996, 1997). (4)(8): The rotation velocity $\left(\mathrm{km} \mathrm{s}^{-1}\right.$ ) at radius $R_{200}$ where the density constrast exceeds 200 (Navarro et al. 1996). (5)(9)(14)(18): Reduced $\chi^{2}$ value. (12)(16): Fitted core-radius of pseudo-isothermal halo model (kpc). (13)(17): Fitted core-density of pseudo-isothermal halo model $\left(10^{-3} M_{\odot} \mathrm{pc}^{-3}\right)$. (..): blank due to unphysically large value or not well-constrained uncertainties. 
Table 4: Parameters of dark halo models for NGC 2366

\begin{tabular}{|c|c|c|c|c|c|c|c|c|}
\hline \multirow[b]{2}{*}{$\Upsilon_{\star} \underset{(1)}{\text { assumption }}$} & \multicolumn{4}{|c|}{ NFW halo (entire region) } & \multicolumn{4}{|c|}{ NFW halo $(<6.0 \mathrm{kpc})$} \\
\hline & $\begin{array}{c}\left\langle\Upsilon_{\star}^{3.6}\right\rangle \\
(2)\end{array}$ & $\begin{array}{c}c \\
(3)\end{array}$ & $\begin{array}{c}V_{200} \\
(4)\end{array}$ & $\begin{array}{c}\chi_{r e d}^{2} \\
(5)\end{array}$ & $\begin{array}{c}\left\langle\Upsilon_{\star}^{3.6}\right\rangle \\
(6)\end{array}$ & $\begin{array}{c}c \\
(7)\end{array}$ & $\begin{array}{c}V_{200} \\
(8)\end{array}$ & $\begin{array}{l}\chi_{\text {red }}^{2} \\
(9)\end{array}$ \\
\hline Min. disk & 0.00 & $<0.1$ & $901.5 \pm 478.4$ & 1.72 & 0.00 & $<0.1$ & $1600.5 \pm \ldots$ & 2.35 \\
\hline Min. disk+gas & 0.00 & $<0.1$ & $727.8 \pm \ldots$ & 1.08 & 0.00 & $<0.1$ & $1136.6 \pm \ldots$ & 1.48 \\
\hline Max. disk & 0.88 & $<0.1$ & $936.1 \pm \ldots$ & 0.89 & 0.88 & $<0.1$ & $954.8 \pm \ldots$ & 1.26 \\
\hline Model $\Upsilon_{*}^{3.6}$ disk & 0.33 & $<0.1$ & $630.7 \pm \ldots$ & 0.98 & 0.33 & $<0.1$ & $1143.6 \pm \ldots$ & 1.37 \\
\hline \multirow[t]{2}{*}{$\Upsilon_{\star}^{\text {free }}$ disk } & 1.15 & $<0.1$ & $665.7 \pm \ldots$ & 1.26 & & & & \\
\hline & \multicolumn{4}{|c|}{ Pseudo-isothermal halo (entire region) } & \multicolumn{4}{|c|}{ Pseudo-isothermal halo $(<6.0 \mathrm{kpc})$} \\
\hline$\Upsilon_{\star} \underset{(10)}{\text { assumption }}$ & $\begin{array}{c}\left\langle\Upsilon_{\star}^{3.6}\right\rangle \\
(11)\end{array}$ & $\begin{array}{l}R_{C} \\
(12)\end{array}$ & $\begin{array}{c}\rho_{0} \\
(13)\end{array}$ & $\begin{array}{l}\chi_{r e d}^{2} \\
(14)\end{array}$ & $\begin{array}{c}\left\langle\Upsilon_{\star}^{3.6}\right\rangle \\
(15)\end{array}$ & $\begin{array}{l}R_{C} \\
(16)\end{array}$ & $\begin{array}{c}\rho_{0} \\
(17)\end{array}$ & $\begin{array}{r}\chi_{r e d}^{2} \\
(18)\end{array}$ \\
\hline Min. disk & 0.00 & $1.47 \pm 0.06$ & $44.6 \pm 2.2$ & 0.16 & 0.00 & $1.49 \pm 0.07$ & $44.1 \pm 2.6$ & 0.21 \\
\hline Min. disk+gas & 0.00 & $1.25 \pm 0.05$ & $43.8 \pm 2.4$ & 0.13 & 0.00 & $1.25 \pm 0.06$ & $43.7 \pm 2.8$ & 0.18 \\
\hline Max. disk & 0.88 & $1.61 \pm 0.15$ & $21.8 \pm 2.5$ & 0.25 & 0.88 & $1.62 \pm 0.18$ & $21.8 \pm 3.0$ & 0.34 \\
\hline Model $\Upsilon_{*}^{3.6}$ disk & 0.33 & $1.36 \pm 0.07$ & $34.8 \pm 2.4$ & 0.17 & 0.33 & $1.36 \pm 0.09$ & $34.7 \pm 2.9$ & 0.23 \\
\hline$\Upsilon_{\star}^{\text {free }}$ disk & -0.99 & $0.98 \pm 0.05$ & $87.1 \pm 14.0$ & 0.14 & & & & \\
\hline
\end{tabular}

Note.-(1)(10): The stellar mass-to-light ratio $\Upsilon_{\star}$ assumptions. "Model $\Upsilon_{\star}^{3.6}$ disk" uses the values derived from the population synthesis models in Section 4.2. $\Upsilon_{\star}^{\text {free }}$ has $\Upsilon_{\star}^{3.6}$ as a free parameter. (2)(6)(11)(15): Average $\Upsilon_{\star}$ in the $3.6 \mu \mathrm{m}$ band $\left(M_{\odot} / L_{\odot}\right)$. (3)(7): Concentration parameter c of NFW halo model (NFW 1996, 1997). (4)(8): The rotation velocity $\left(\mathrm{km} \mathrm{s}^{-1}\right.$ ) at radius $R_{200}$ where the density constrast exceeds 200 (Navarro et al. 1996). (5)(9)(14)(18): Reduced $\chi^{2}$ value. (12)(16): Fitted core-radius of pseudo-isothermal halo model (kpc). (13)(17): Fitted core-density of pseudo-isothermal halo model $\left(10^{-3} M_{\odot} \mathrm{pc}^{-3}\right)$. (..): blank due to unphysically large value or not well-constrained uncertainties. 


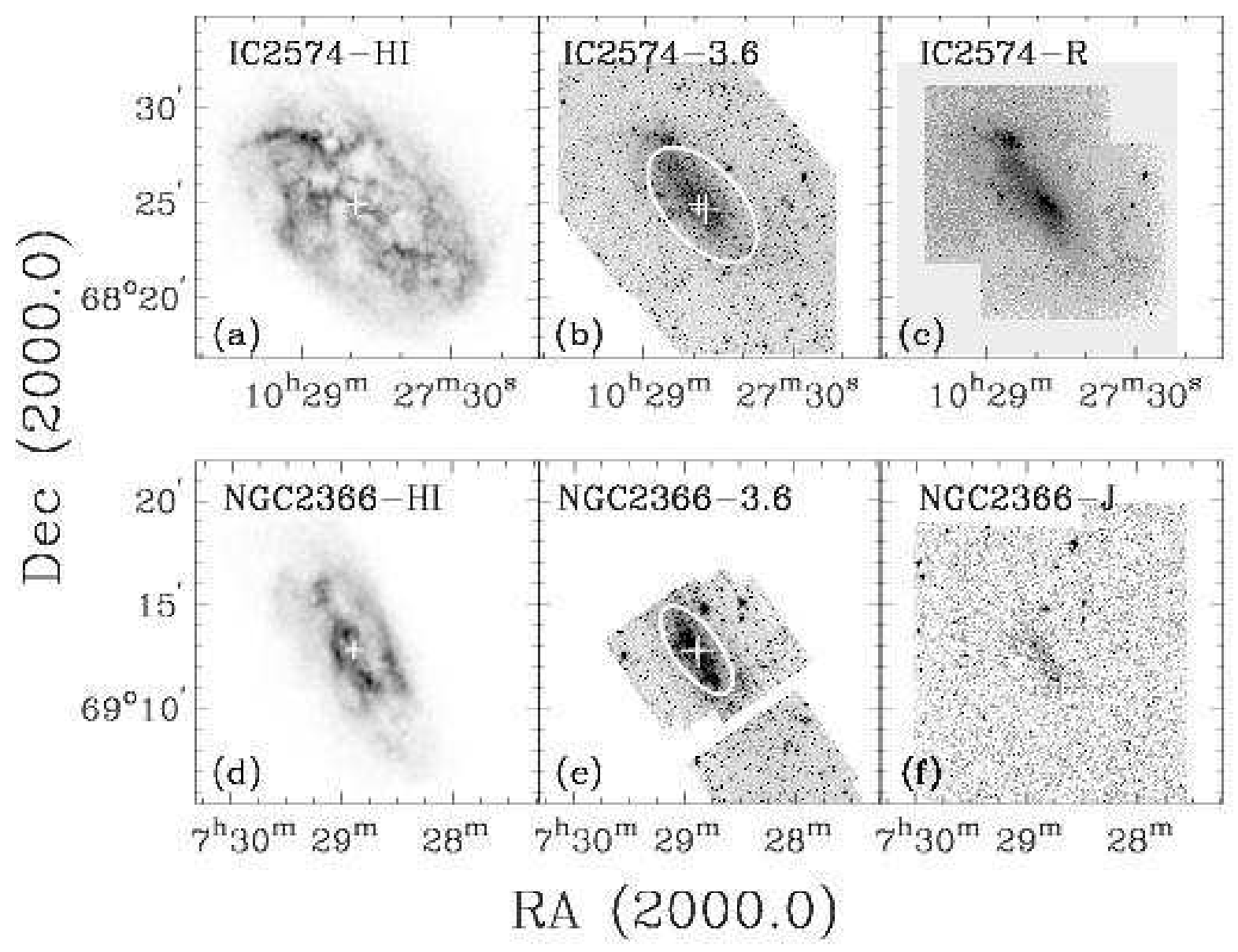

Fig. 1.- Total intensity maps of IC 2574 and NGC 2366 in various bands. (a)(d): Integrated THINGS Hi maps. The crosses indicate the derived dynamical centers in this paper. (b)(e): 3.6 $\mu \mathrm{m}$ image with superimposed the photometric centers as listed in NED (large crosses) and the dynamical centers as derived in this paper from the bulk velocity fields (small crosses). The ellipses indicate the ellipse fit discussed in Section 3.1. (c): Optical $R$-band image obtained with the $2.1 \mathrm{~m}$ telescope at KPNO as part of the ancillary SINGS data (Kennicutt et al. 2003). (f): $J$-band image from 2MASS. 


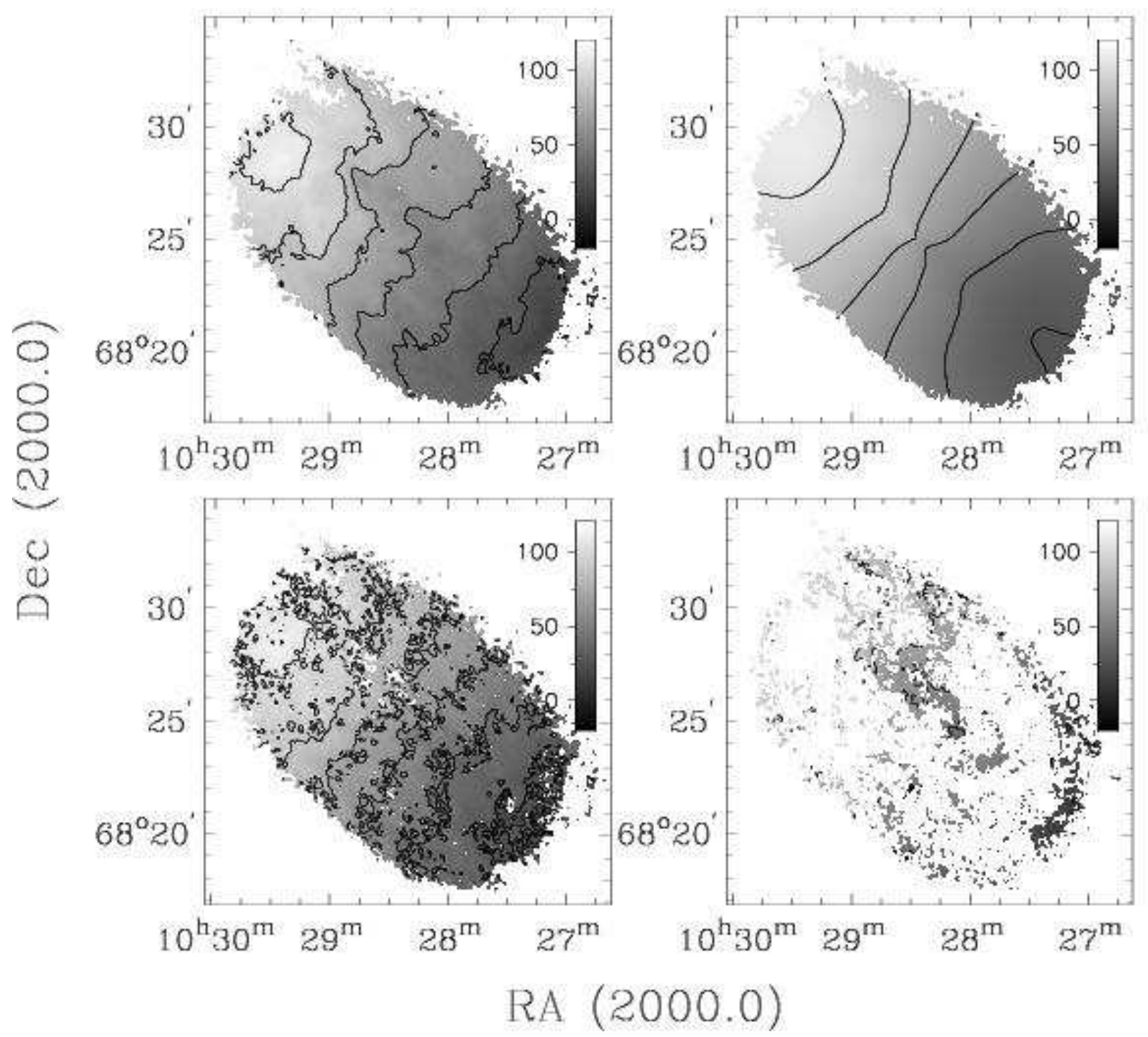

Fig. 2.- The velocity fields of IC 2574. Top-left: Intensity-weighted mean (IWM) velocity field from THINGS. Top-right: Initial value velocity field for the bulk motion. Bottom-left: Bulk velocity field. Bottom-right: Velocity field of the non-circular motions. Velocity contours run from $-60 \mathrm{~km} \mathrm{~s}^{-1}$ to $120 \mathrm{~km} \mathrm{~s}^{-1}$ with a spacing of $20 \mathrm{~km} \mathrm{~s}^{-1}$. Note that the strongest non-circular motions seen in the central region of the IWM velocity field are removed in the extracted bulk velocity field. See Section 3.1 for a full description. 


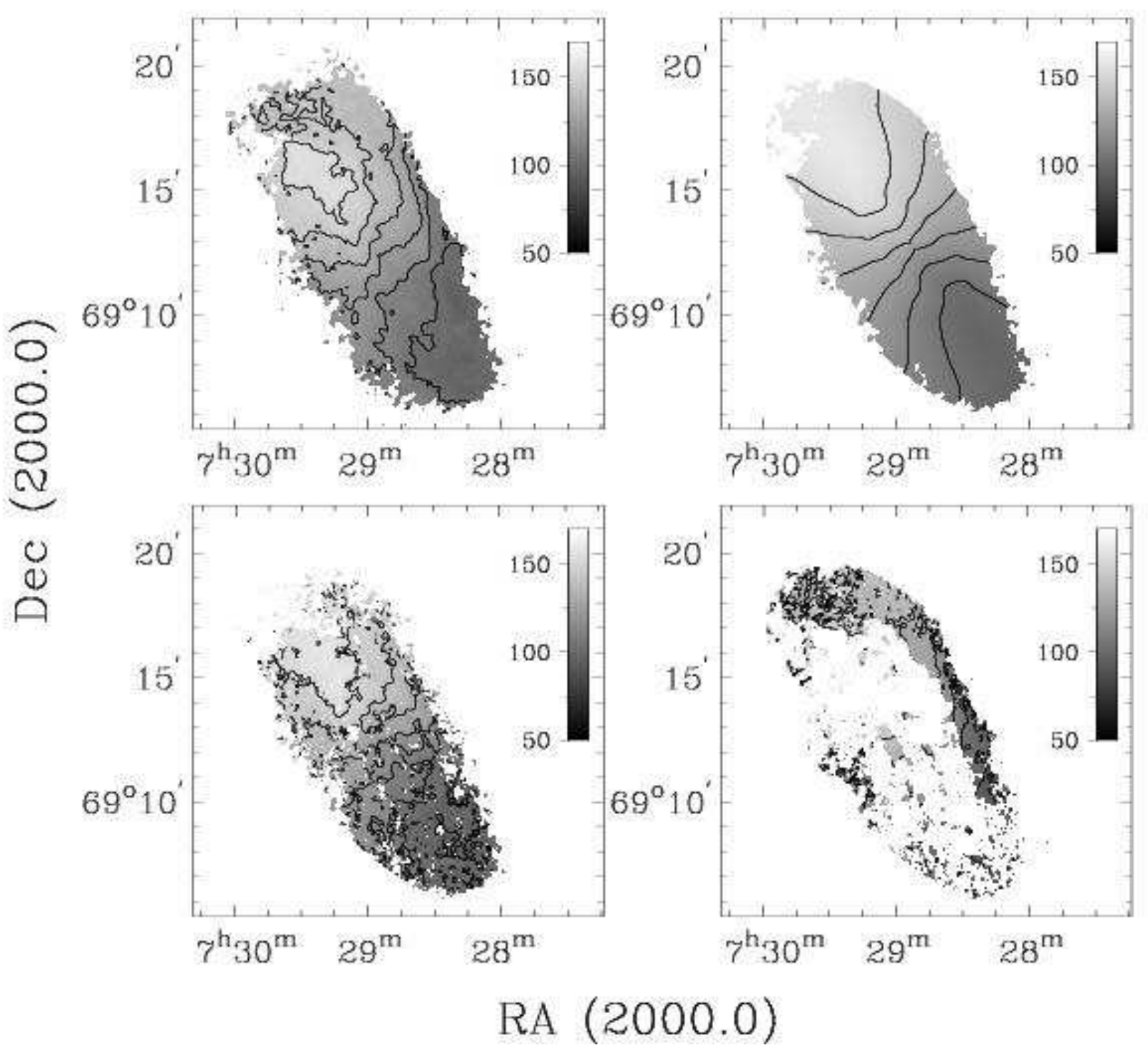

Fig. 3.- The velocity fields of NGC 2366. Top-left: Intensity-weighted mean (IWM) velocity field from THINGS. Top-right: Initial value velocity field for the bulk motion. Bottom-left: Bulk velocity field. Bottom-right: Velocity field of the non-circular motions. Velocity contours run from $-20 \mathrm{~km} \mathrm{~s}^{-1}$ to $170 \mathrm{~km} \mathrm{~s}^{-1}$ with a spacing of $15 \mathrm{~km} \mathrm{~s}^{-1}$. Note that the strongest non-circular motions are found in the outer part. See Section 3.1 for a full description. 

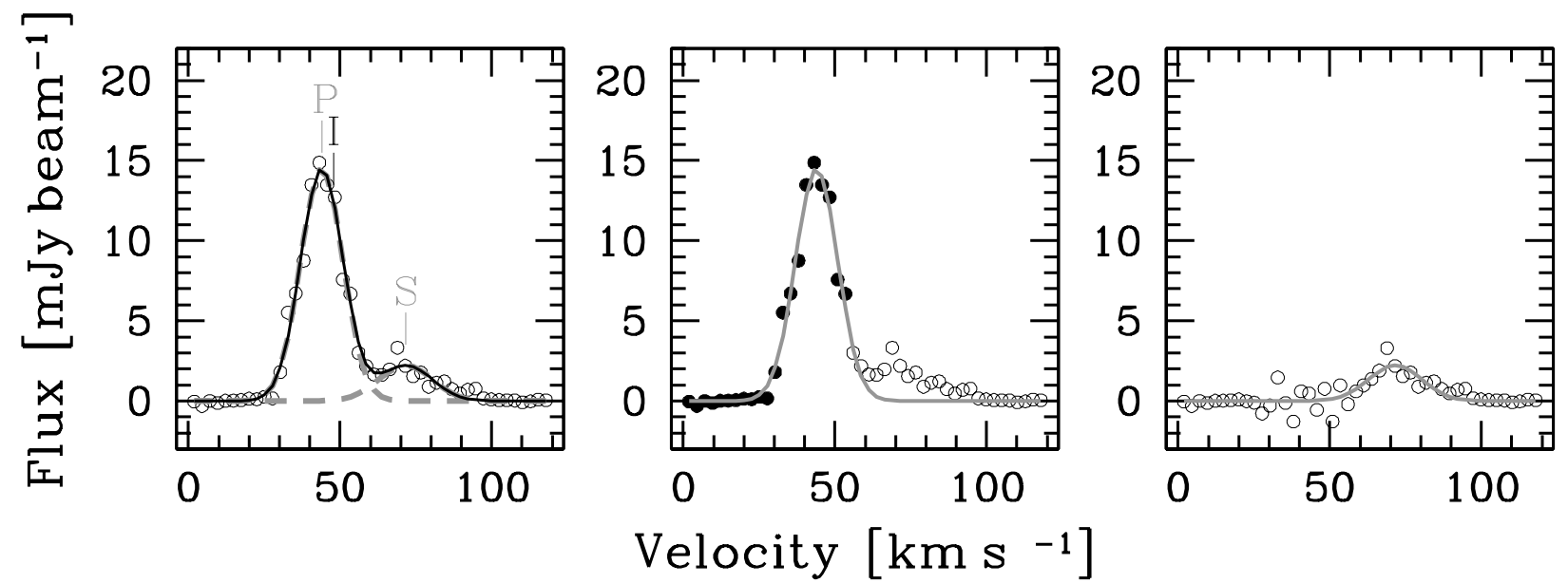

Fig. 4.- A schematic example of Gaussian decomposition using two Gaussian functions. Left: Decomposed profiles with two Gaussian functions. P and S represent the central values of the primary and secondary Gaussian components and I denotes the IWM value. Middle: The primary Gaussian component is fitted only to the data points that are unaffected by the secondary component (filled circles). Right: The secondary Gaussian component is fitted to the residual after subtracting the primary Gaussian component from the raw profile. Note that the IWM value will deviate from that of the primary component by an amount which depends on the significance of the secondary. Section 3.1 gives a more detailed description. 


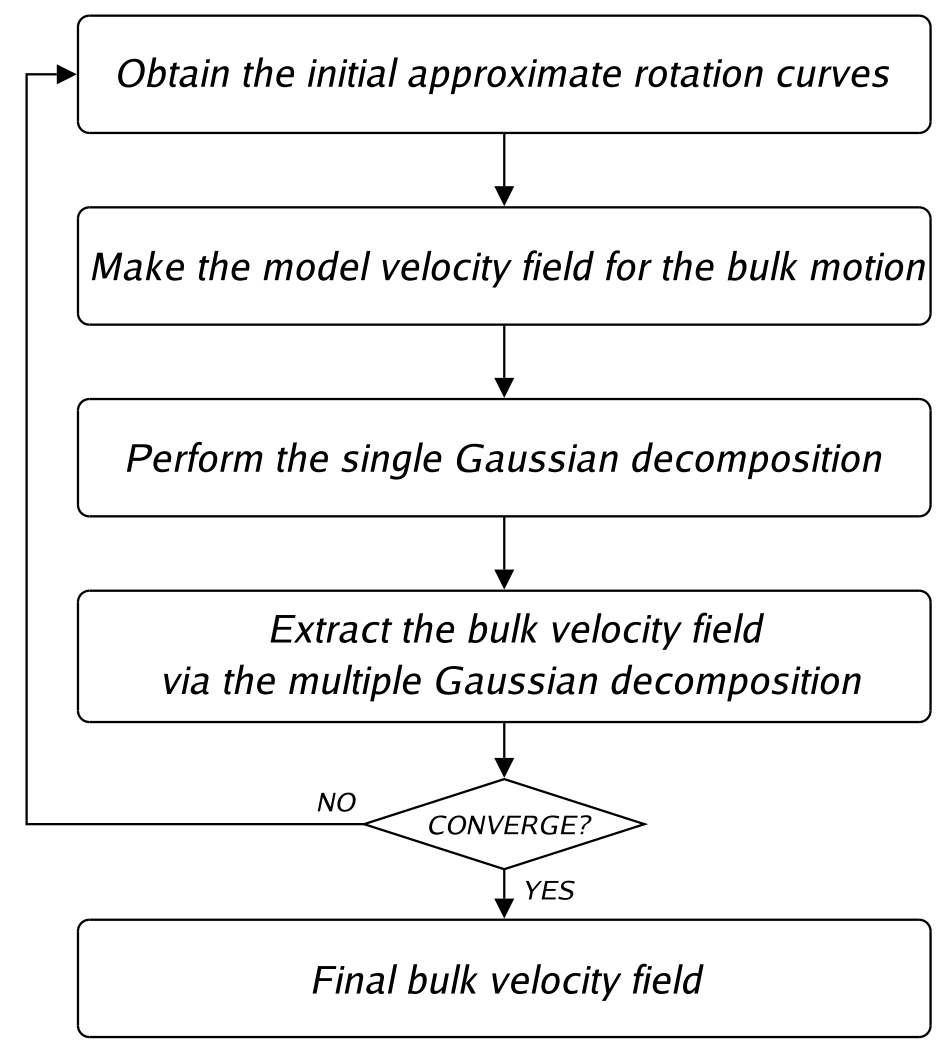

Fig. 5.- A schematic diagram of the bulk-motion extraction method. This flow chart shows the overall procedure for extracting the bulk velocity field from the Hi data cube. The procedure is iterated until the convergence criterion is met (see text for details). The method is fully described in Section 3.1. 


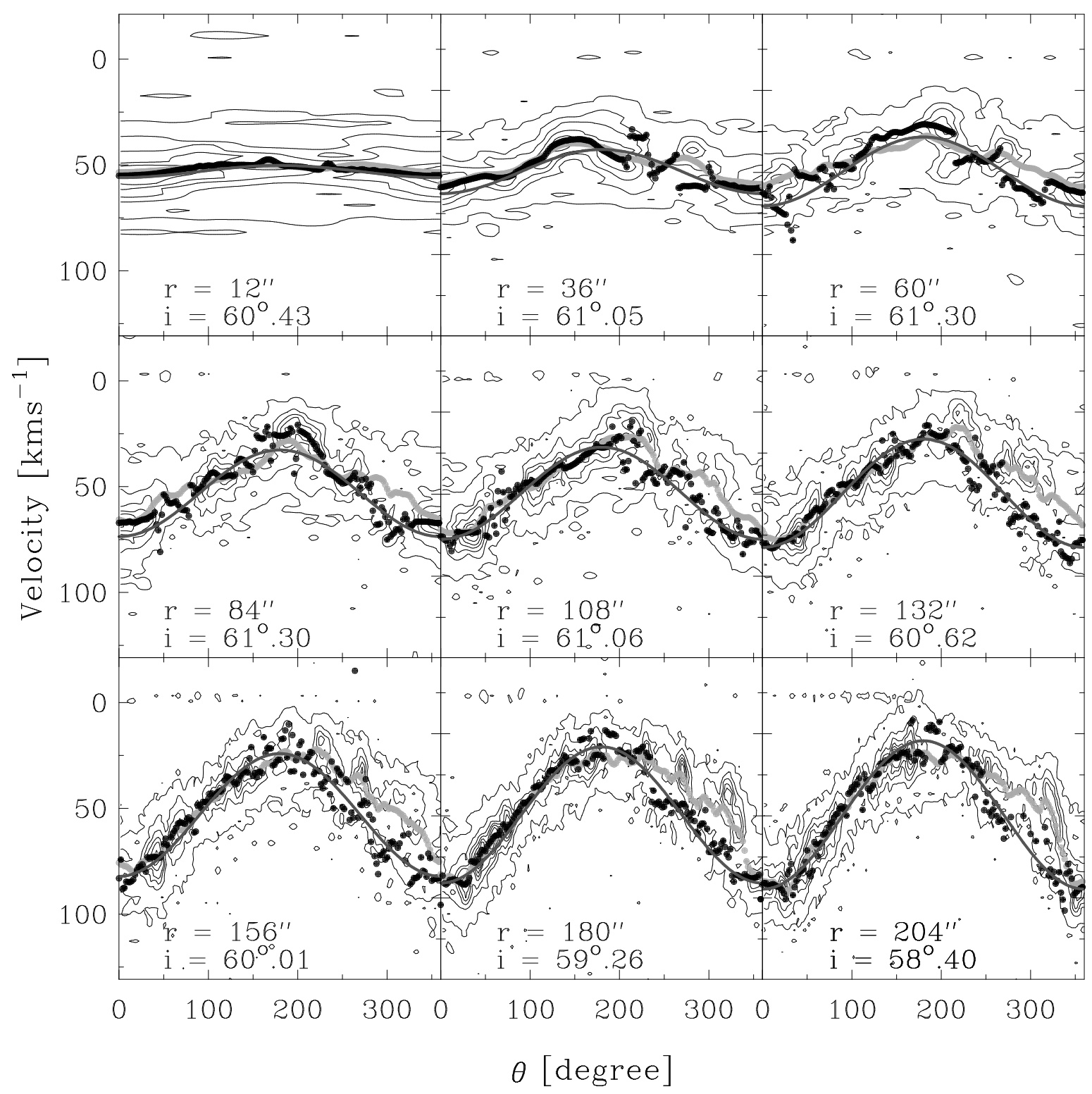

Fig. 6.- Azimuthal position-velocity diagrams of IC 2574. The observed velocities are displayed as a function of $\theta$ along ellipses in the plane of sky. The angle $\theta$ is measured anticlockwise with respect to the receding major axis in the plane of the sky. Each ellipse is defined by a radius and inclination as labeled in each panel. All ellipses have identical dynamical centers as derived in this paper from the bulk velocity field (see Fig. 1). The width of the ellipse rings used for the azimuthal integration is $1.5^{\prime \prime}$. Contours start at $+1 \sigma$ in steps of $+5 \sigma$ with $\sigma=0.56$ mJy beam $^{-1}$. The light-gray dots represent the IWM velocity and the black dots indicate the bulk velocity derived by our method in Section 3.3. The dark-gray solid line on each panel represents the expected "bulk" motion. In many cases, especially for $R<300^{\prime \prime}$ and $\theta \sim 320^{\circ}$, the IWM velocities deviate significantly from the symmetric shape of the rotation curve. 


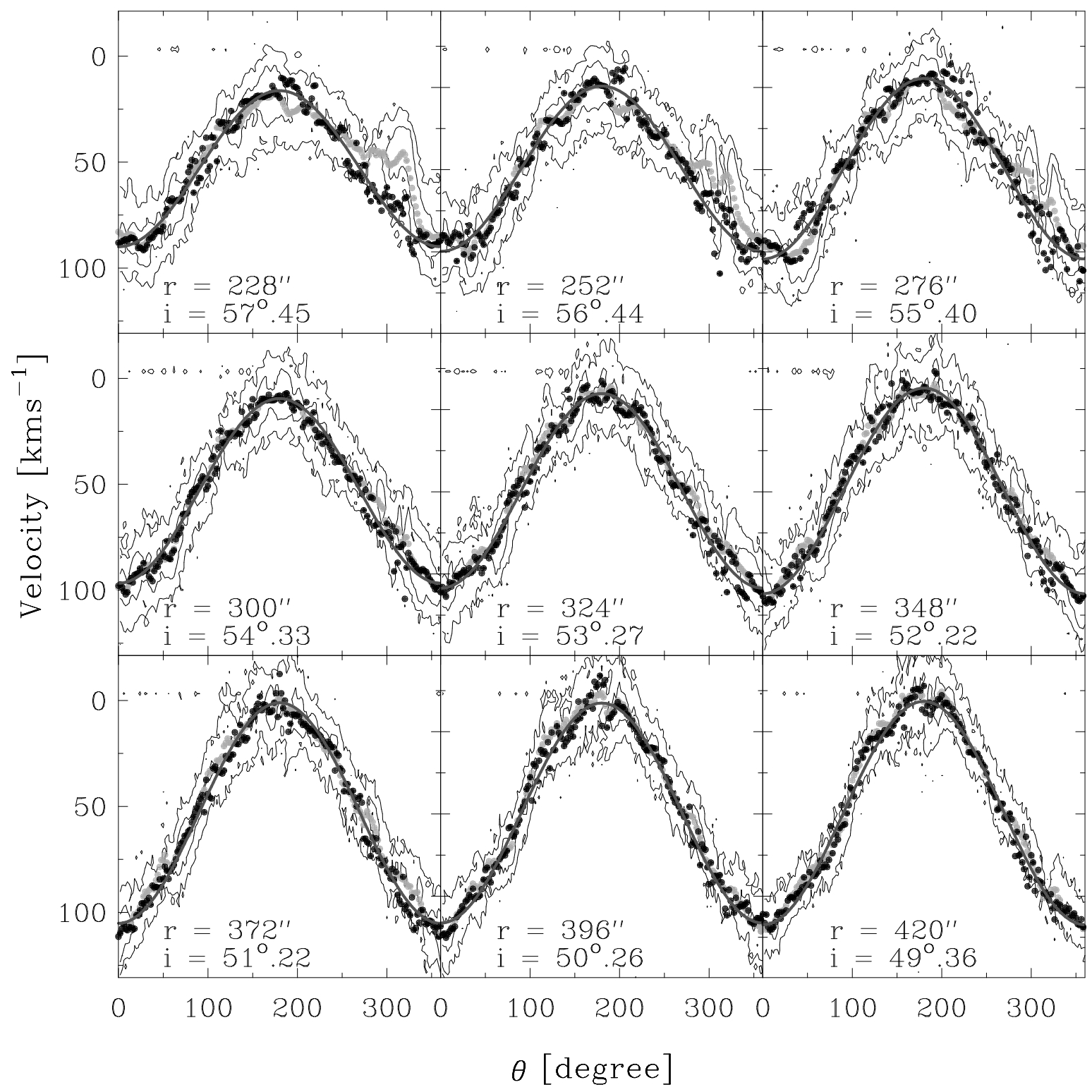

Fig. 6.- Position-velocity diagrams of IC 2574 (cont'd). 


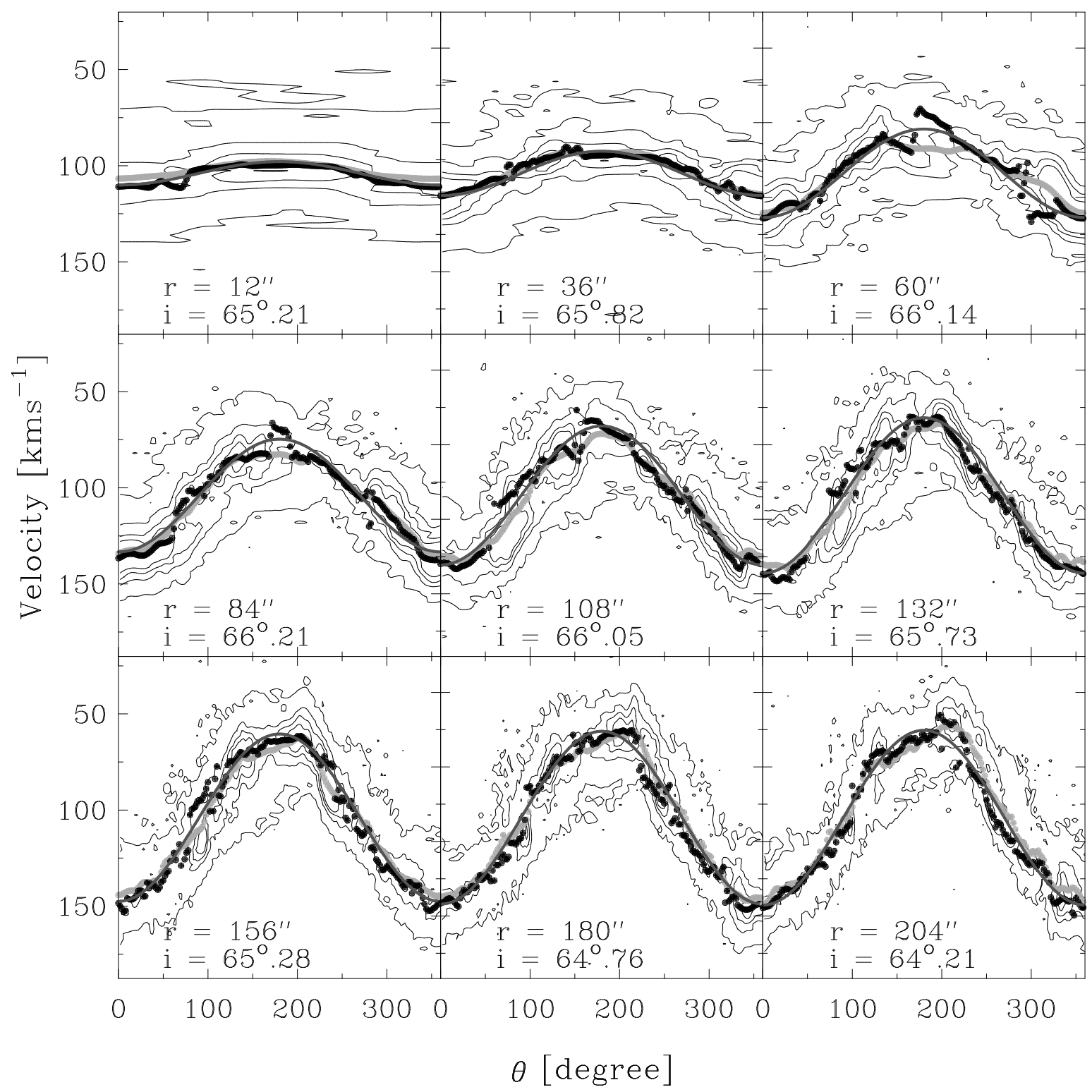

Fig. 7.- Azimuthal position-velocity diagrams of NGC 2366. The observed velocities are displayed as a function of $\theta$ along ellipses in the plane of sky. The angle $\theta$ is measured anticlockwise with respect to the receding major axis in the plane of the sky. Each ellipse is defined with a radius and inclination as labeled in each panel. All ellipses have identical dynamical centers as derived in this paper from the bulk velocity field (see Fig. 1). The width of the ellipse rings used for the azimuthal integration is $1.5^{\prime \prime}$. Contours start at $+2 \sigma$ in steps of $+10 \sigma$ with $\sigma=0.52$ mJy beam $^{-1}$. The light-gray dots represent the IWM velocity and the black dots indicate the bulk velocity derived by our method in Section 3.3. The dark-gray solid line on each panel represents the expected "bulk" motion. In many cases, especially for $R>300^{\prime \prime}$ and $\theta \sim 270^{\circ}$, the IWM velocities deviate significantly from the symmetric shape of the rotation curve. 


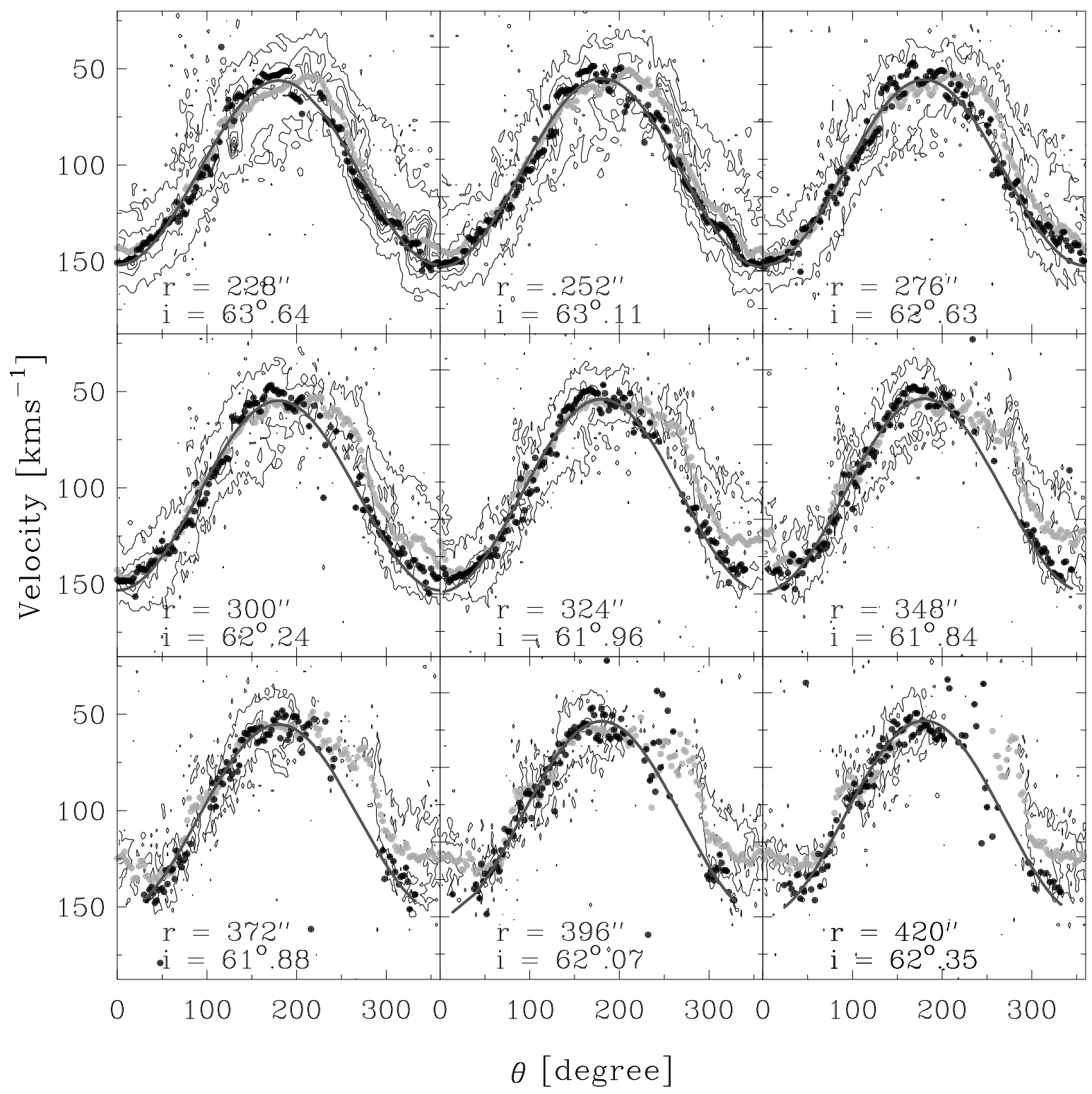

Fig. 7.- Position-velocity diagrams of NGC 2366 (cont'd). 

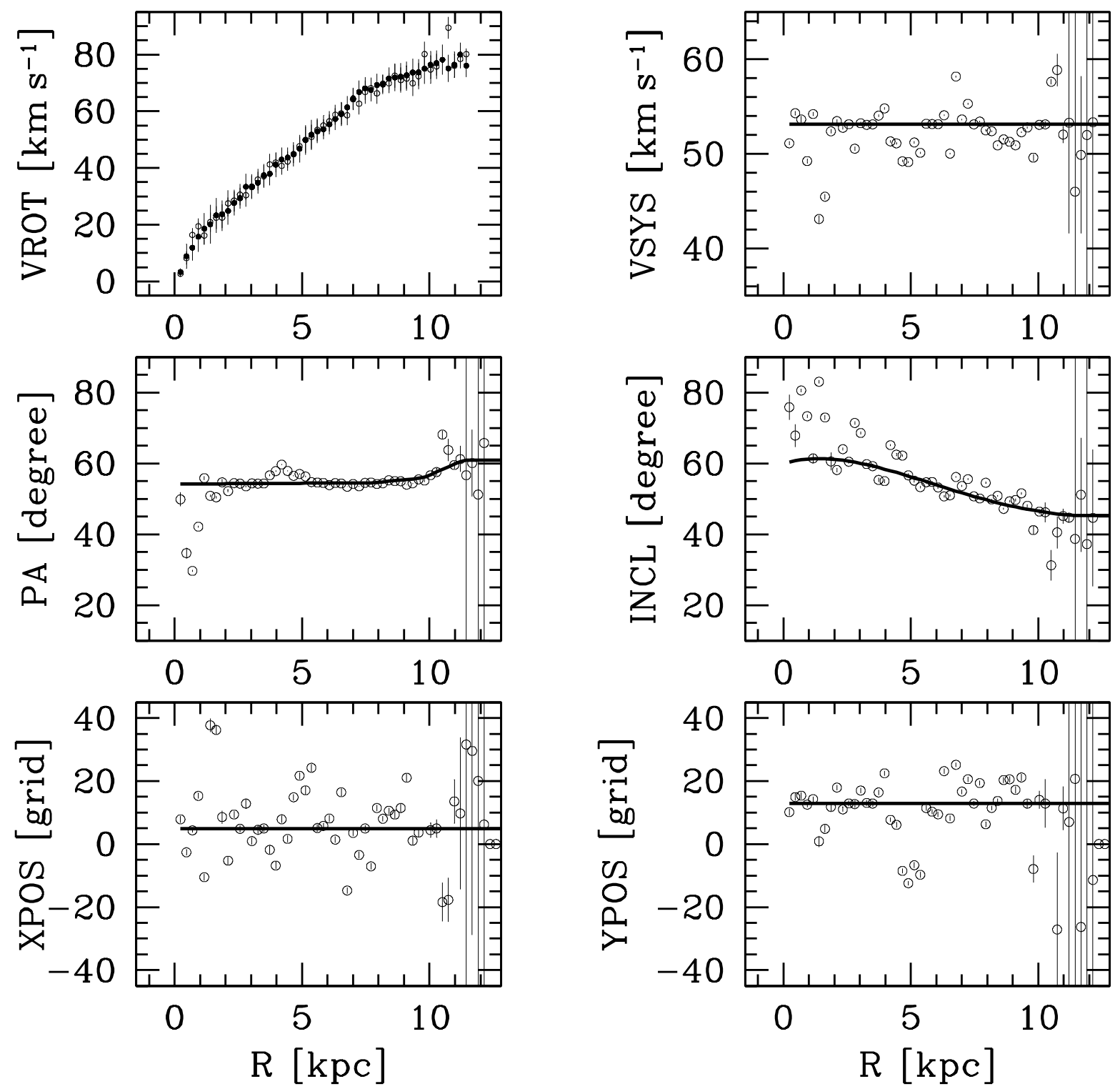

Fig. 8. - The tilted ring model derived from the bulk velocity field of IC 2574. The open black circles in all panels indicate the fit made with all parameters free. The filled black circles in the VROT panel were derived using the entire velocity field after fixing other ring parameters to the values (solid lines) as shown in the panels. See Fig. 11 for a larger scale. In all other panels the solid lines show the values as a function of radius adopted for the final tilted ring model. 

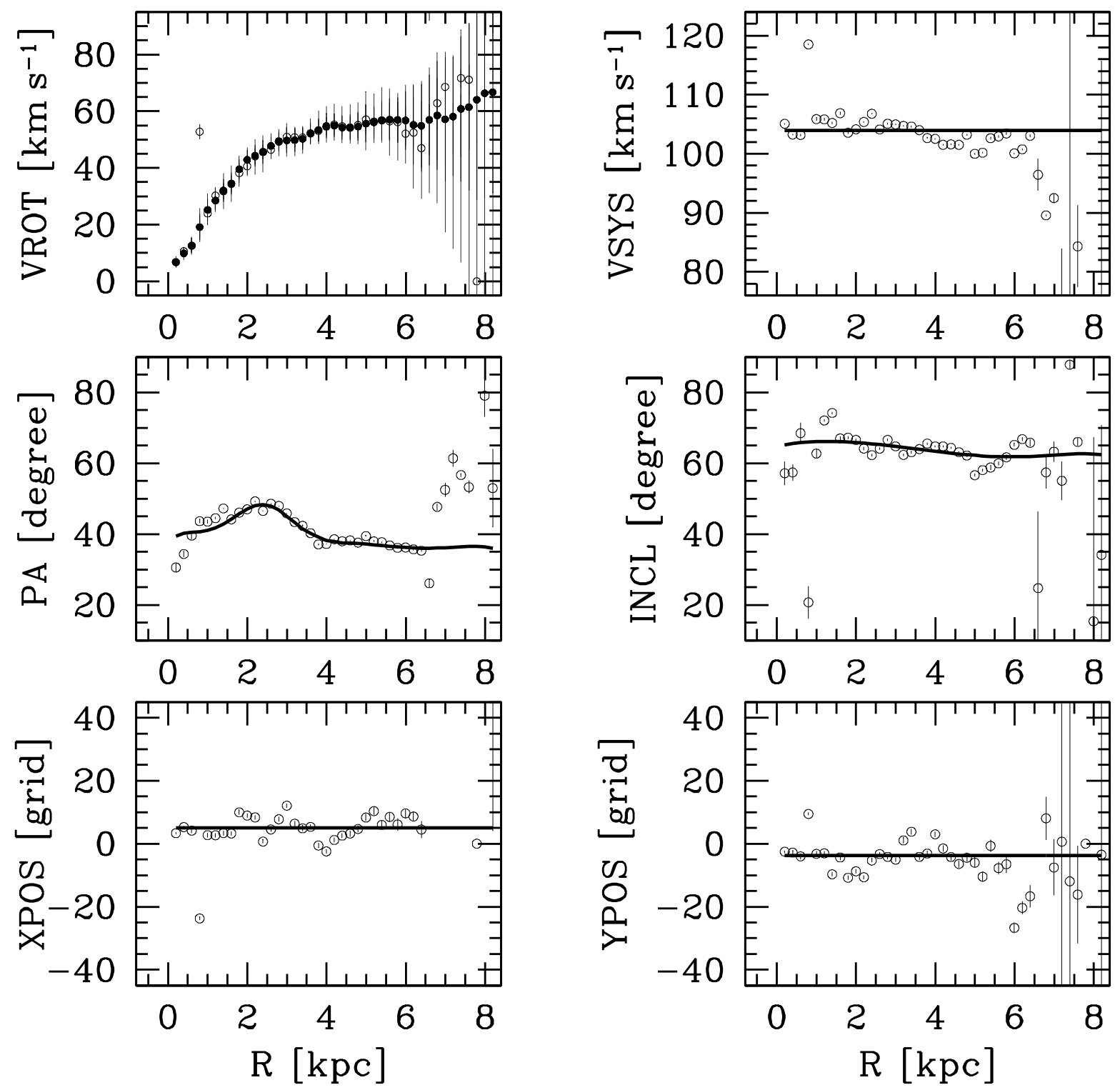

Fig. 9.- The tilted ring model derived from the bulk velocity field of NGC 2366. The open black circles in all panels indicate the fit made with all ring parameters free. The filled black circles in the VROT panel were derived using the entire velocity field after fixing other ring parameters to the values (solid lines) as shown in the panels. See Fig. 13 for a larger scale. In all other panels the solid lines show the values as a function of radius adopted for the final tilted ring model. 

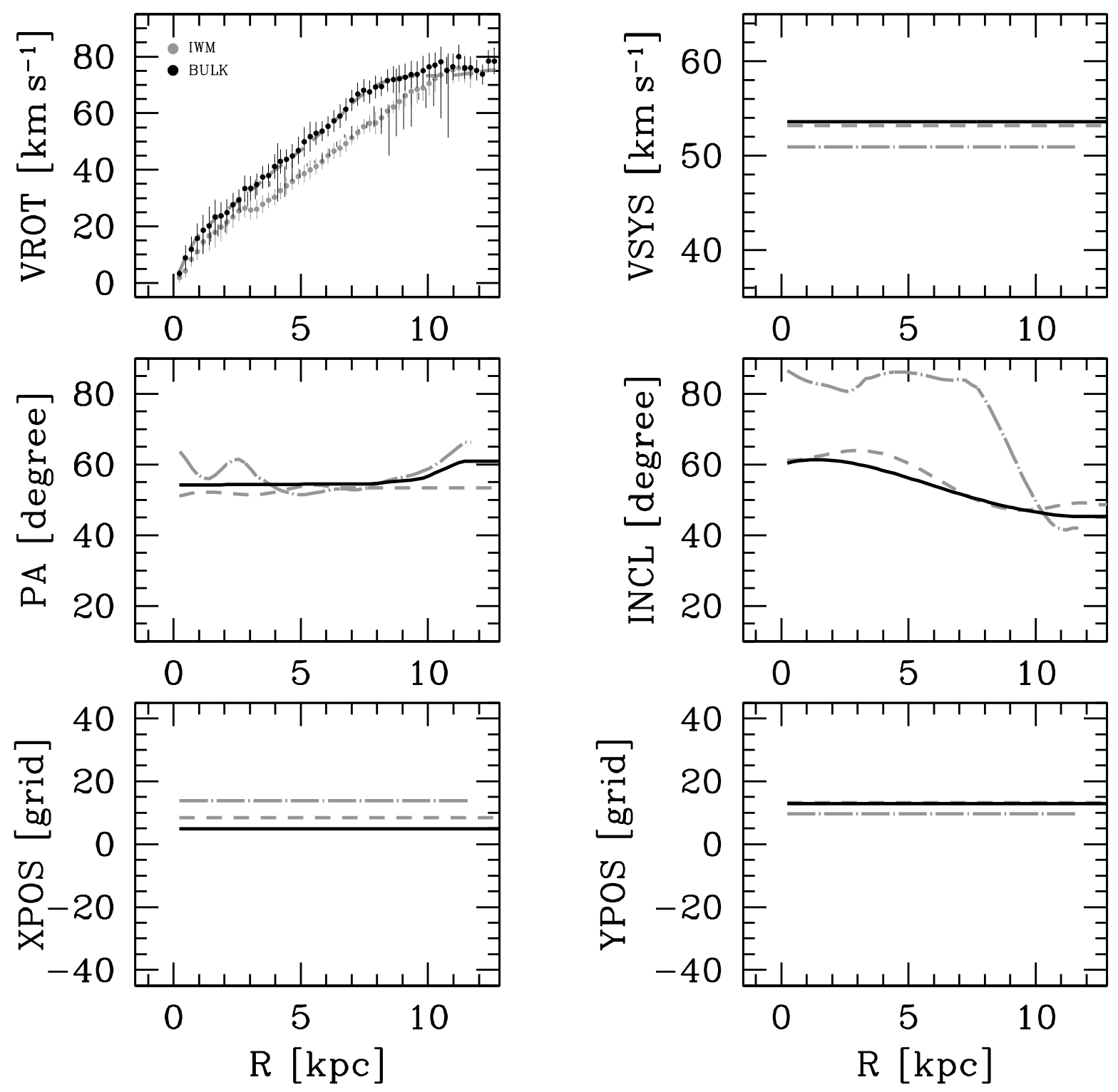

Fig. 10.- Comparison of the HI rotation curves derived from the IWM and bulk velocity fields of IC 2574. The filled gray circles and long dash-dotted lines represent the rotation curves from the IWM velocity field. The gray dashed lines are used as initial condition for a bulk velocity field model. The black dots and solid lines show the adopted rotation curves of IC 2574 using the bulk velocity field. The large difference in inclinations between IWM and bulk velocity fields is clearly evident in the panel of INCL (inclination) and this results in a significant difference $\left(\sim 14 \mathrm{~km} \mathrm{~s}^{-1}\right)$ of VROT (rotation velocity). More details are given in Section 3.3. 


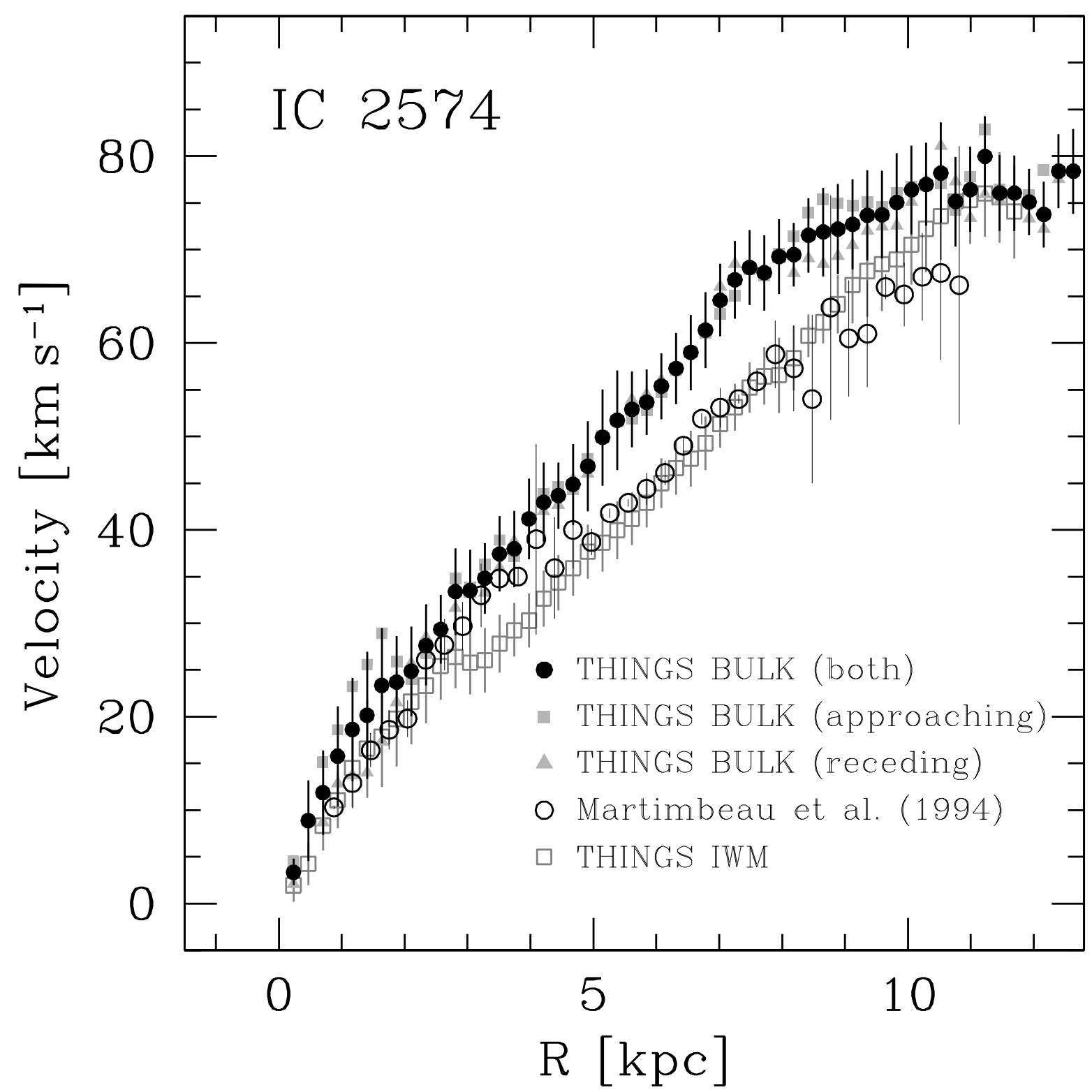

Fig. 11. - Comparison of the rotation curve of IC 2574 with the rotation curves from literature. The filled black circles and open squares represent the rotation curves from the bulk and IWM velocity field, respectively. The filled gray squares and triangles are derived using only the approaching and receding sides of the bulk velocity field with the final tilted ring model in Fig. 8 (black solid curves). Open circles were adopted from Martimbeau et al. (1994). More details are given in Section 3.3. 

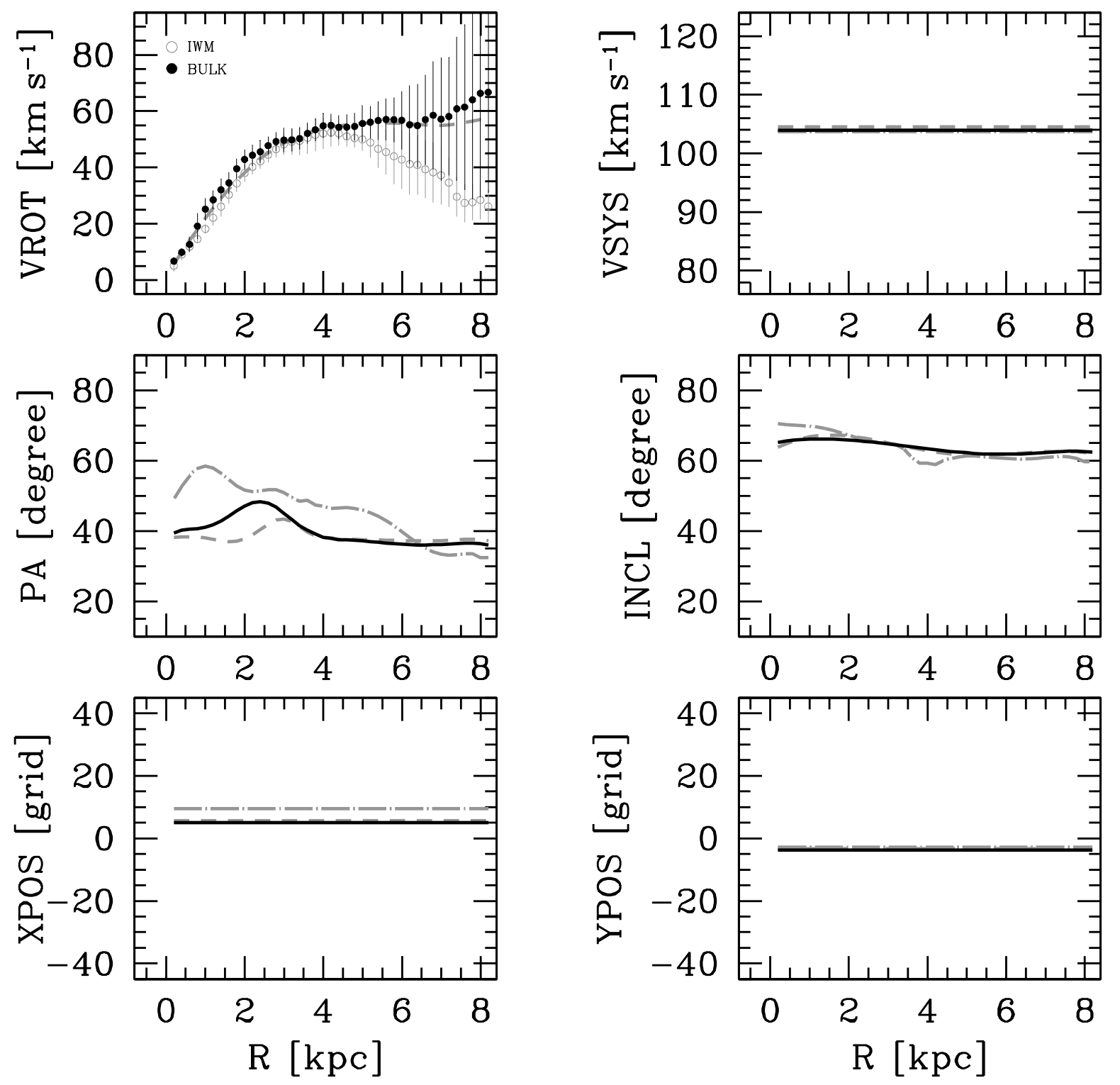

Fig. 12. - Comparison of the HI rotation curves derived from the IWM and bulk velocity fields of NGC 2366. The open gray circles and long dash-dotted lines represent the rotation curves from the IWM velocity field. The gray dashed lines are used as initial condition for a bulk velocity field model. The black dots and solid lines show the adopted rotation curves of NGC 2366 using the bulk velocity field. See Fig. 13 for a detailed comparison with literature values. 


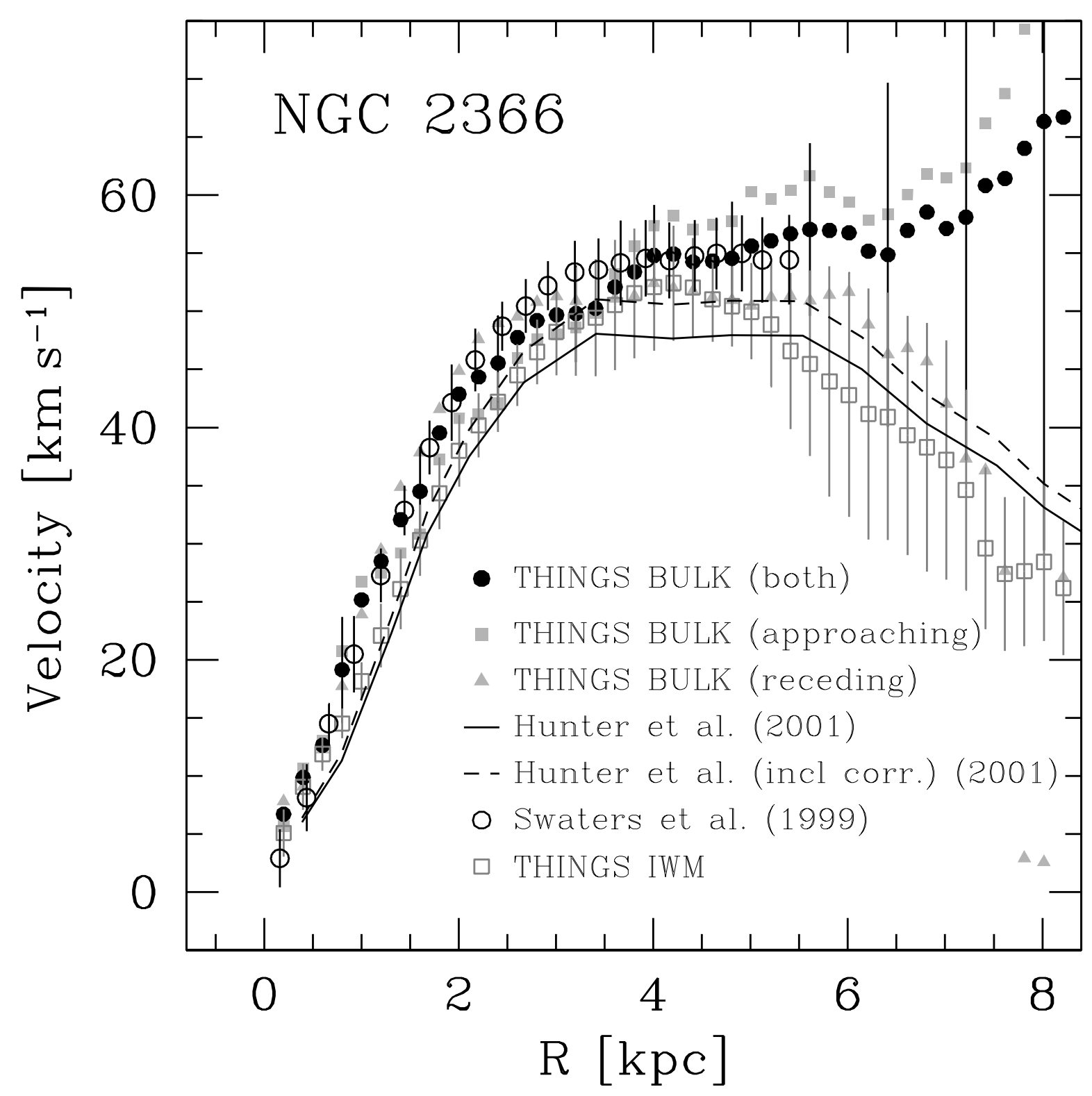

Fig. 13. - Comparison of the rotation curve of NGC 2366 with rotation curves from the literature. The filled black circles and open squares represent the rotation curves from the bulk and IWM velocity field, respectively. The filled gray squares and triangles are derived using only the approaching and receding sides of the bulk velocity field with the final tilted ring model in Fig. 9 (black solid curves). Open circles were adopted from Swaters et al. (1999) and the solid line shows the rotation curve from Hunter et al. (2001). The dashed line indicates the corrected Hunter et al. (2001) curve using the inclination of the THINGS IWM curve. A full description is given in Section 3.3. 

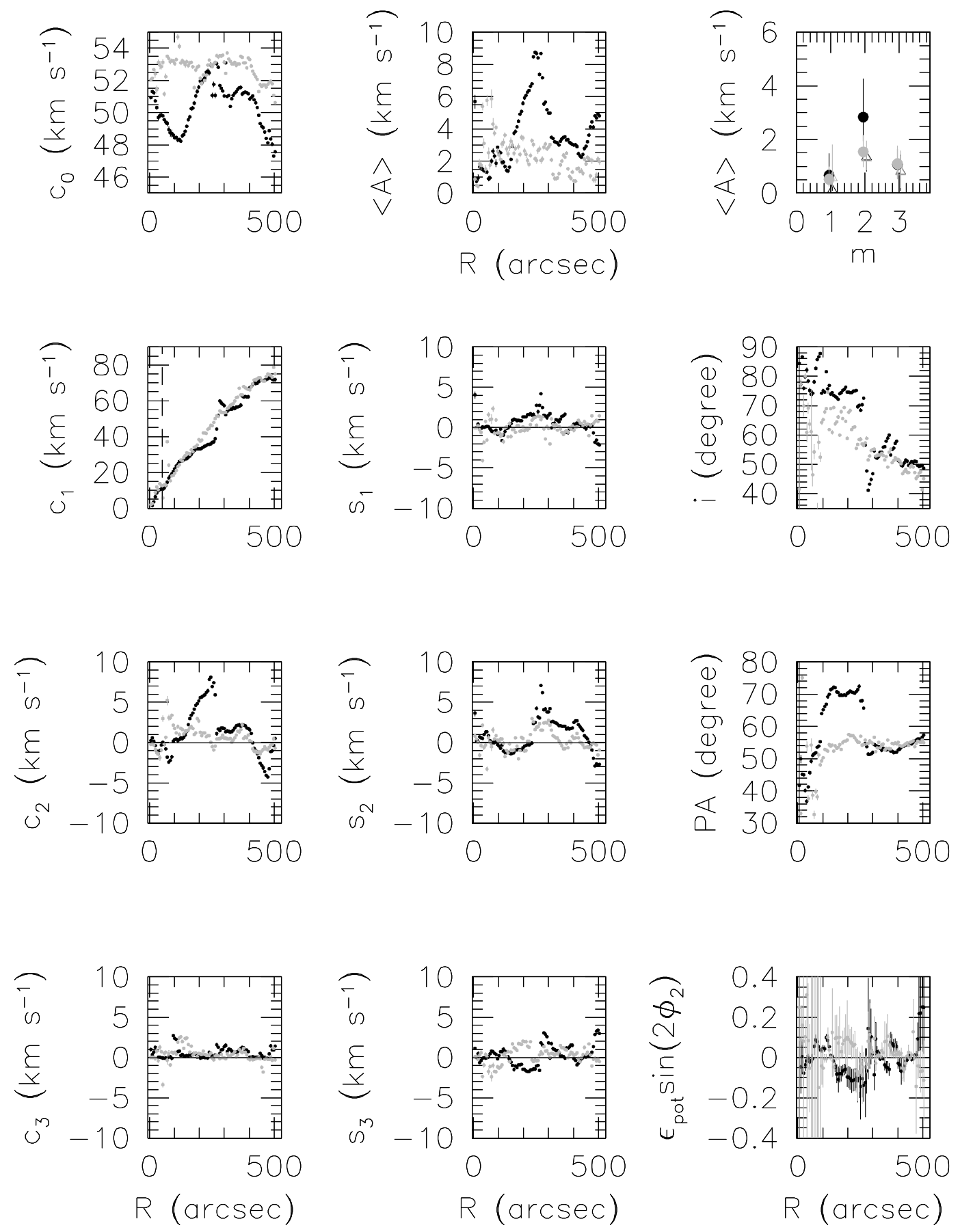

Fig. 14.- Harmonic expansion for IC 2574. $c_{0}$ and $c_{1}$ are the systemic and the rotation velocities, respectively. $c_{2}, c_{3}, s_{1}, s_{2}$, and $s_{3}$ components quantify non-circular motions. $\langle A\rangle$ is the median absolute amplitudes as given in Eq. 2, 3, and 4. Black and gray dots represent the harmonic decomposition results of the IWM velocity field and the bulk velocity, respectively. See Section 3.3 for a detailed description. 

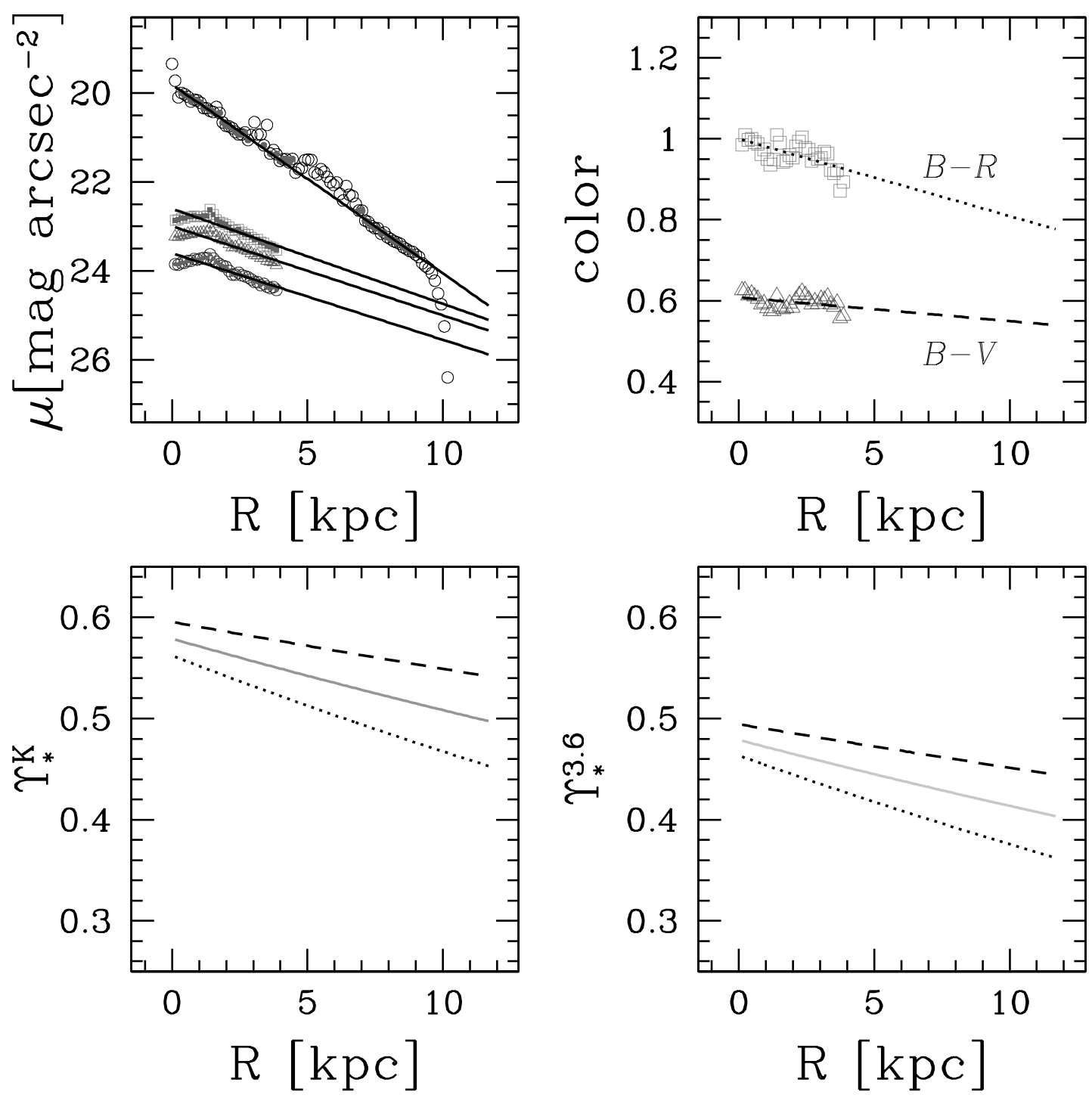

Fig. 15.- Top-left: Azimuthally averaged surface brightness profiles (not corrected for inclination) of IC 2574 in the $3.6 \mu \mathrm{m}, R, V$, and $B$ bands (top to bottom) derived assuming the tilted-ring parameters shown in Fig. 8. The curves shown are fitted to the data at $R<8 \mathrm{kpc}$ for $3.6 \mu \mathrm{m}$ and at $R<4 \mathrm{kpc}$ for $B, V$, and $R$ bands (partly filled points). Top-right: Radial color profiles of IC 2574. Dotted line and dashed line are for fits to the $B-R$ and $B-V$ colors, respectively. Bottom-left: Radial variation of the model $\Upsilon_{\star}$ in the $K$-band of IC 2574 . The dotted and dashed lines are computed using optical colors $(B-R$ and $B-V)$ and the mean value (solid line) is adopted as the final model $\Upsilon_{\star}$ in the $K$-band. The relationships between $\Upsilon_{\star}^{K}$ and optical colors (e.g., $B-R, B-V$ ) are adopted from the models of Bell \& de Jong (2001). Bottom-right: model $\Upsilon_{\star}$ in the $3.6 \mu \mathrm{m}$ band of IC 2574. Eq. 6 is used for converting $\Upsilon_{\star}^{K}$ to $\Upsilon_{\star}^{3.6}$ (see Section 4.2 for details). The average values of $\Upsilon_{\star}^{3.6}$ from different $\Upsilon_{\star}$ assumptions for IC 2574 are presented in Table 3. 


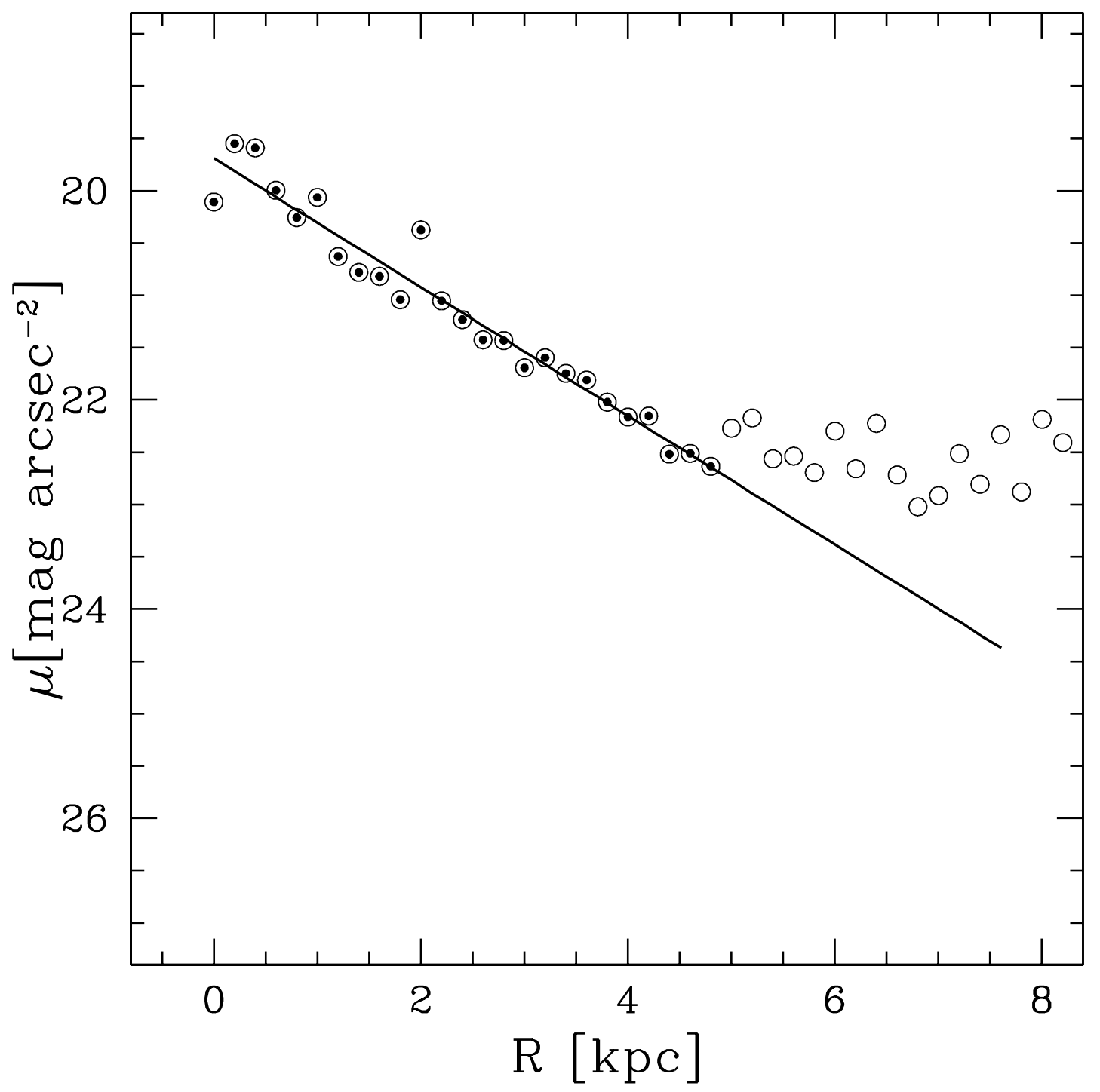

Fig. 16. - Azimuthally averaged surface brightness profile (not corrected for inclination) of NGC 2366 in the $3.6 \mu \mathrm{m}$ derived assuming the tilted-ring parameters shown in Fig. 9. The curve shown is fitted to the data at $R<5 \mathrm{kpc}$ (partly filled circles). 

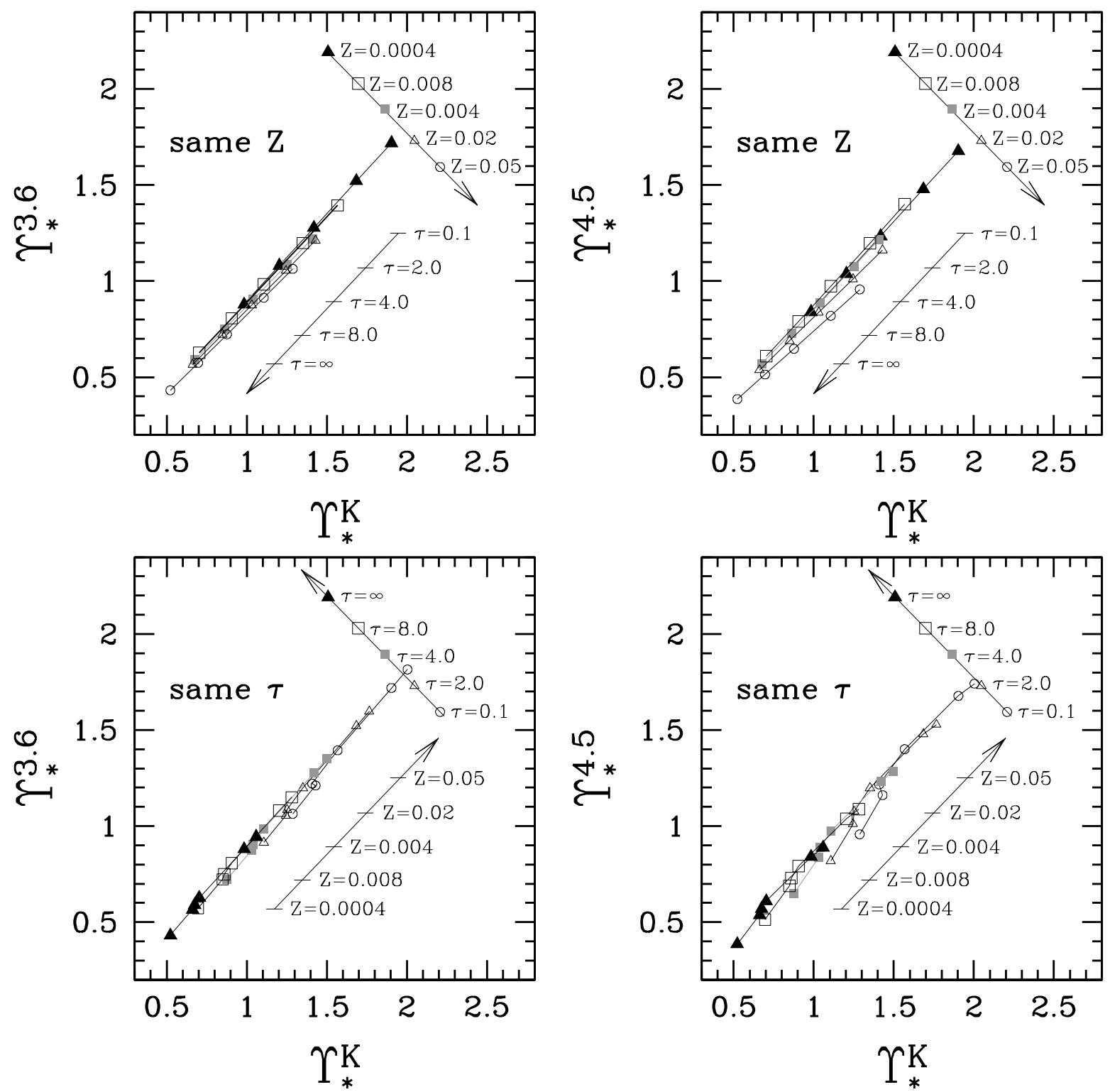

Fig. 17. - The relations between the $\Upsilon_{\star}^{K}$ and the $\Upsilon_{\star}$ in the $3.6 \mu \mathrm{m}$ and $4.5 \mu \mathrm{m}$ bands. Metallicity is parameterized by the parameter $Z$ and $\tau$ represents the $e$-folding time-scale for the exponentially declining star formation (Bruzual 1983). Upper panels: Models of the same metallicity $Z$ but different $\tau$ are connected by lines. $\tau$ ranges from 0.1 Gyr to $\infty$ as indicated by arrows. $Z$ varies with $0.0004,0.004,0.008,0.02$, and 0.05 as illustrated by different symbols. Lower panels: Models of the same $\tau$ but different metallicity $Z$ are connected by lines. $Z$ ranges from 0.0004 to 0.05 as indicated by arrows. $\tau$ varies with $0.1,2,4,8 \mathrm{Gyr}$, and $\infty$ as illustrated by different symbols. 

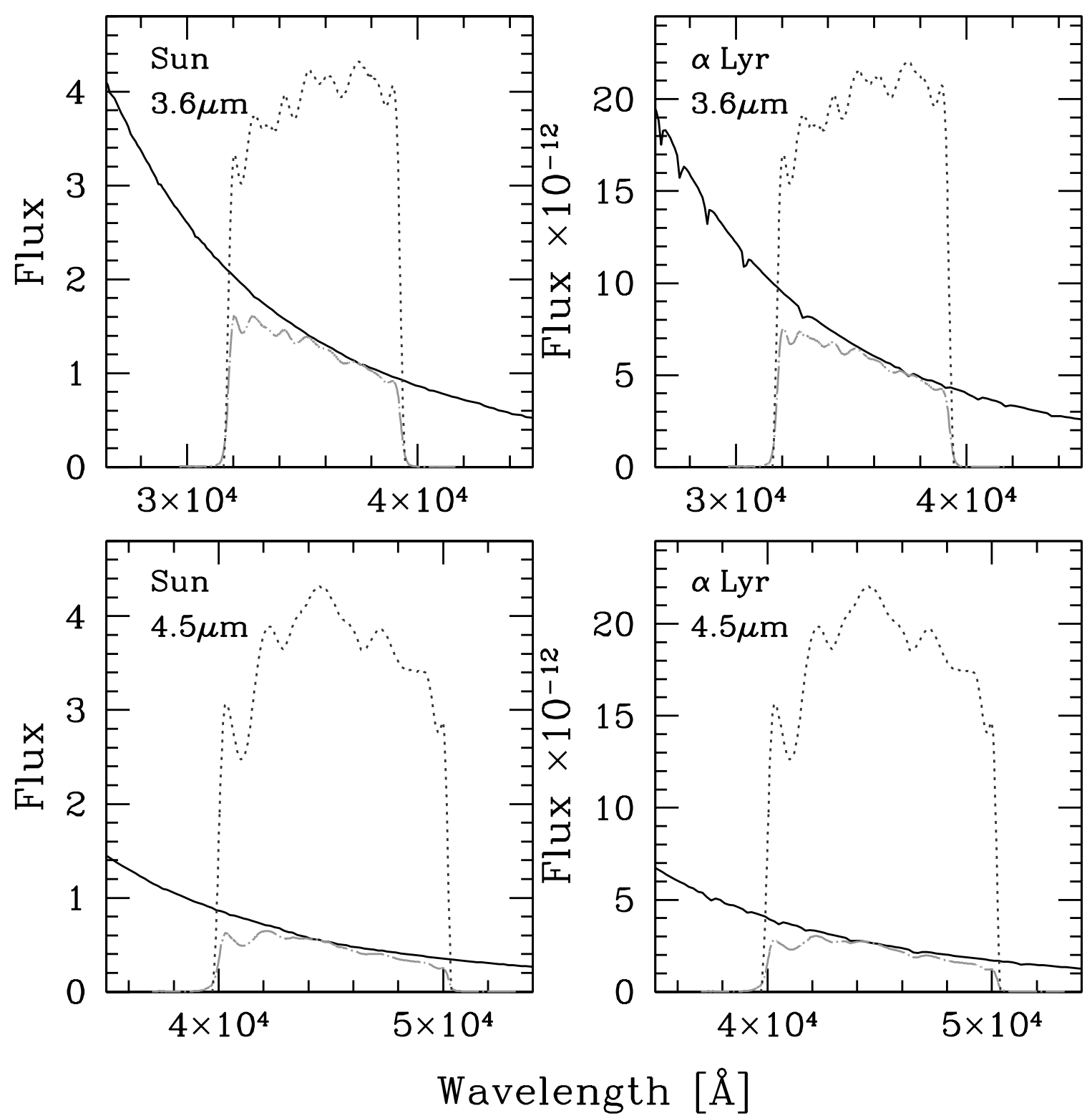

Fig. 18. - The spectral energy distribution of the Sun and $\alpha$ Lyr convolved with the IRAC filter

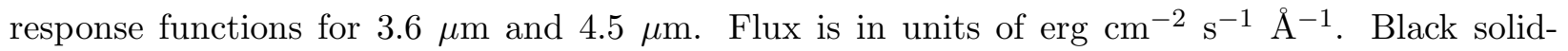
lines: Spectral energy distribution of the Sun and $\alpha$ Lyr. Dotted lines: filter response functions of the IRAC bands $(3.6 \mu \mathrm{m}$ and $4.5 \mu \mathrm{m})$. Dash-dotted lines denote the convolved spectral energy distribution of the Sun and $\alpha$ Lyr. 

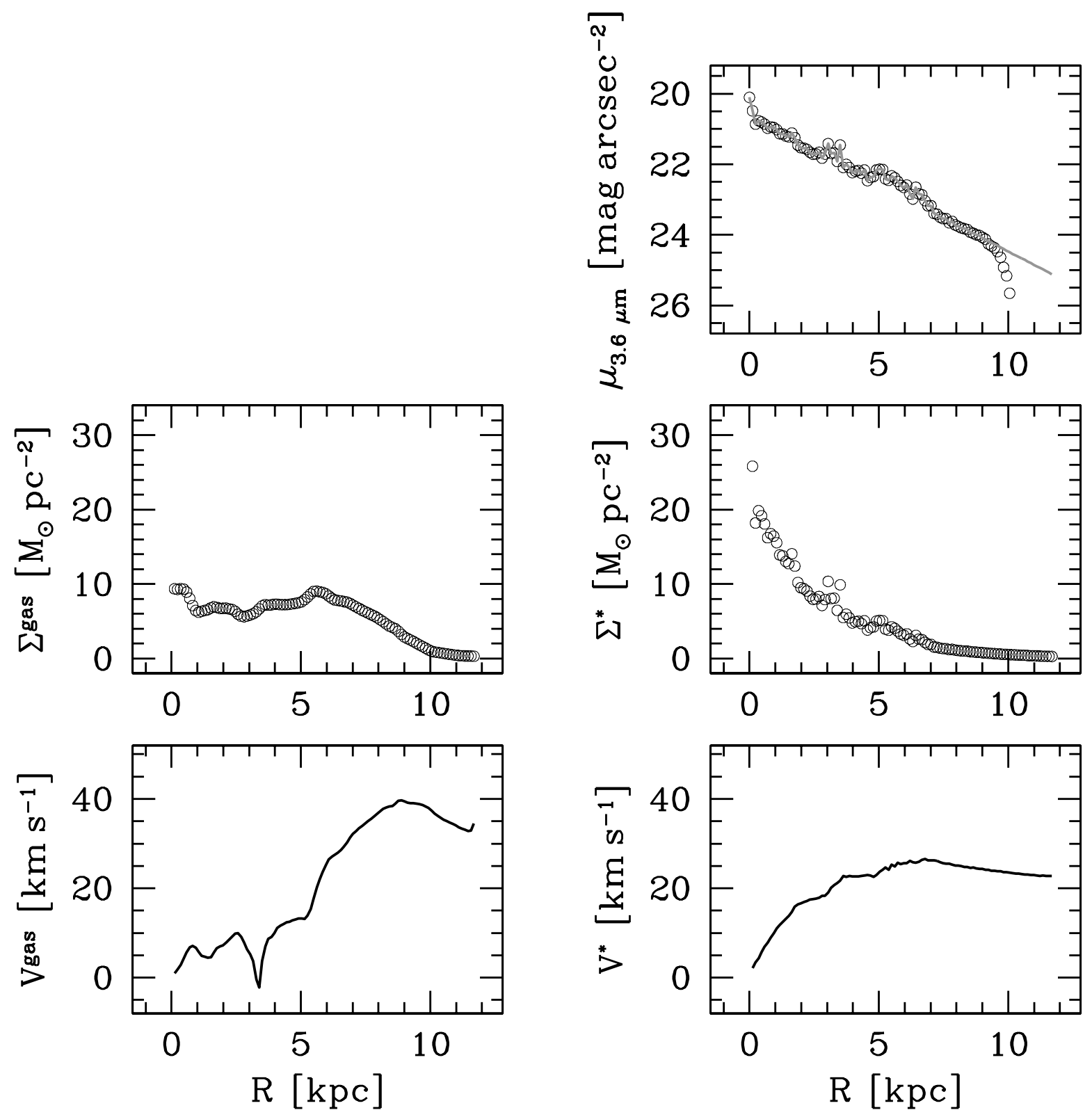

Fig. 19. - Mass models for the gas and stellar components of IC 2574. The left two panels show the radial mass surface density distribution of neutral gas (observed Hi scaled by 1.4 to account for He and metals) and corresponding rotation curve derived from this. The three panels on the right from top to bottom represent the radial surface brightness (inclination corrected) in the 3.6 $\mu \mathrm{m}$ band, stellar mass surface density, and rotation velocity of the disk using the model $\Upsilon_{\star}^{3.6}$ values shown in Fig. 15. The radial average of the model $\Upsilon_{\star}^{3.6}$ values is given in Table 3 . 

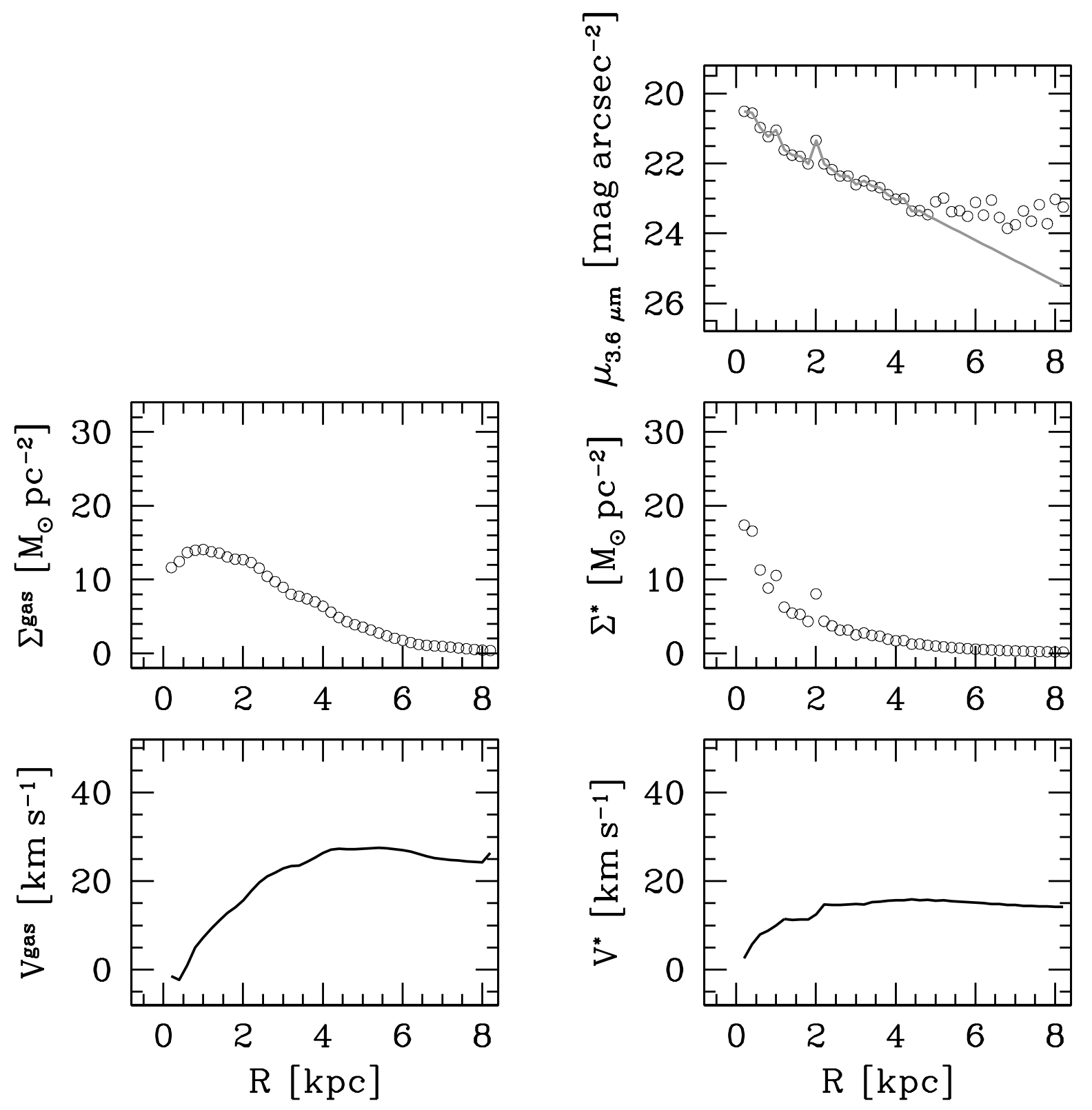

Fig. 20. - Mass models for the gas and stellar components of NGC 2366. The left two panels show the radial mass surface density distribution of neutral gas (observed Hi scaled by 1.4 to account for He and metals) and corresponding rotation curve derived from this. The three panels on the right from top to bottom represent the radial surface brightness (inclination corrected) in the 3.6 $\mu \mathrm{m}$ band, stellar mass surface density, and rotation velocity of the disk using the model $\Upsilon_{\star}^{3.6}$ values given in Table 2 . 

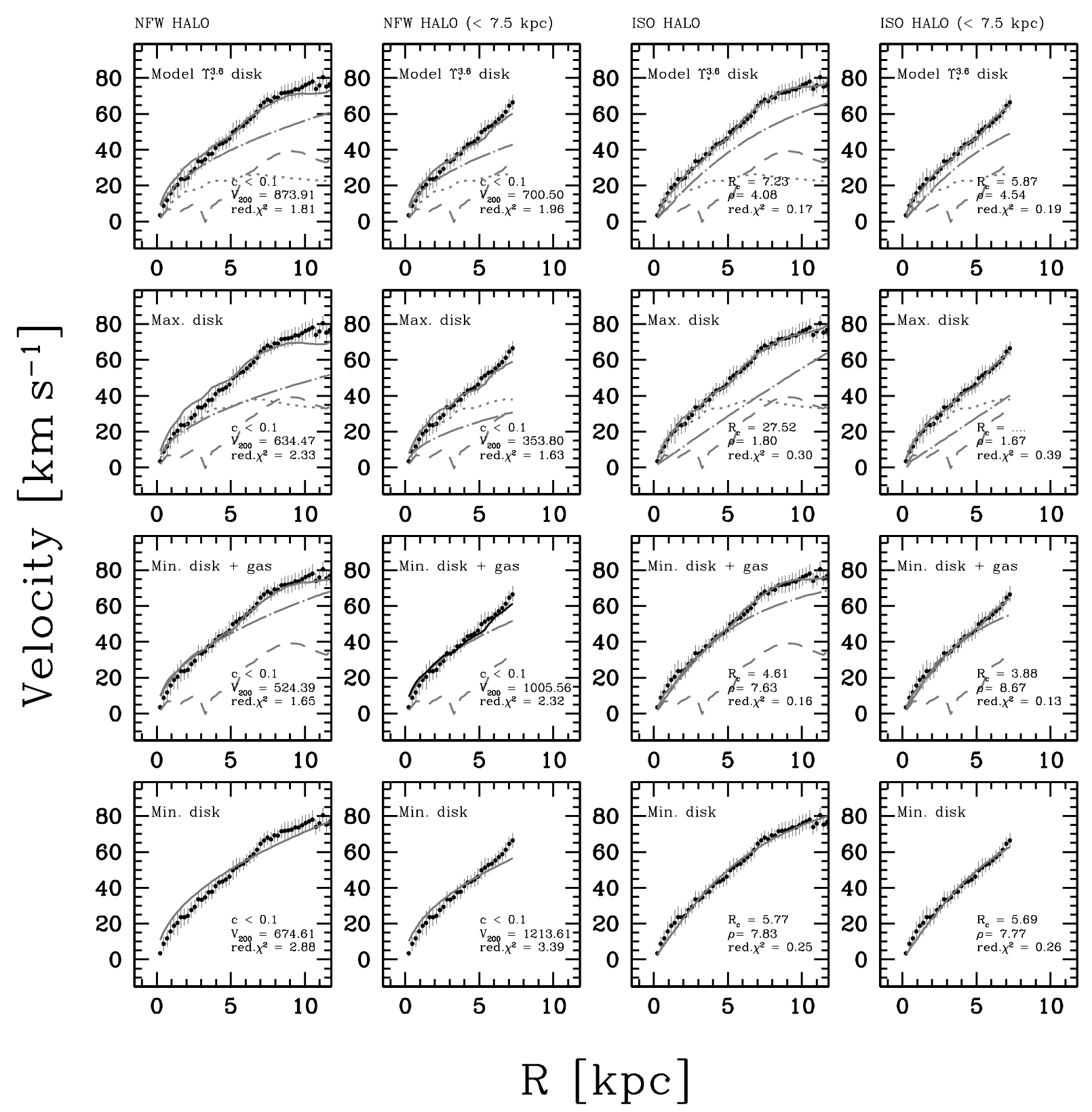

Fig. 21.- Disk-halo decomposition of the IC 2574 rotation curve under various $\Upsilon_{\star}$ assumptions (model $\Upsilon_{\star}^{3.6}$, maximum disk, minimum disk + gas, and minimum disk). The long dashed lines represent the rotation curves of the gas component; the short dashed lines are the rotation curves of the stellar disk; the long dash-dotted lines the rotation curves of the dark matter halo. The dots are the observed rotation curves from the bulk velocity field while the full lines are the sum of all contributions. The fitted parameters for each halo model (NFW and pseudo-isothermal dark matter halo models) are denoted on each panel along with the reduced $\chi^{2}$ value. Note that the pseudo-isothermal halo gives much better fits to the observed curves than the NFW halo. This is discussed more fully in Section 5.2. 

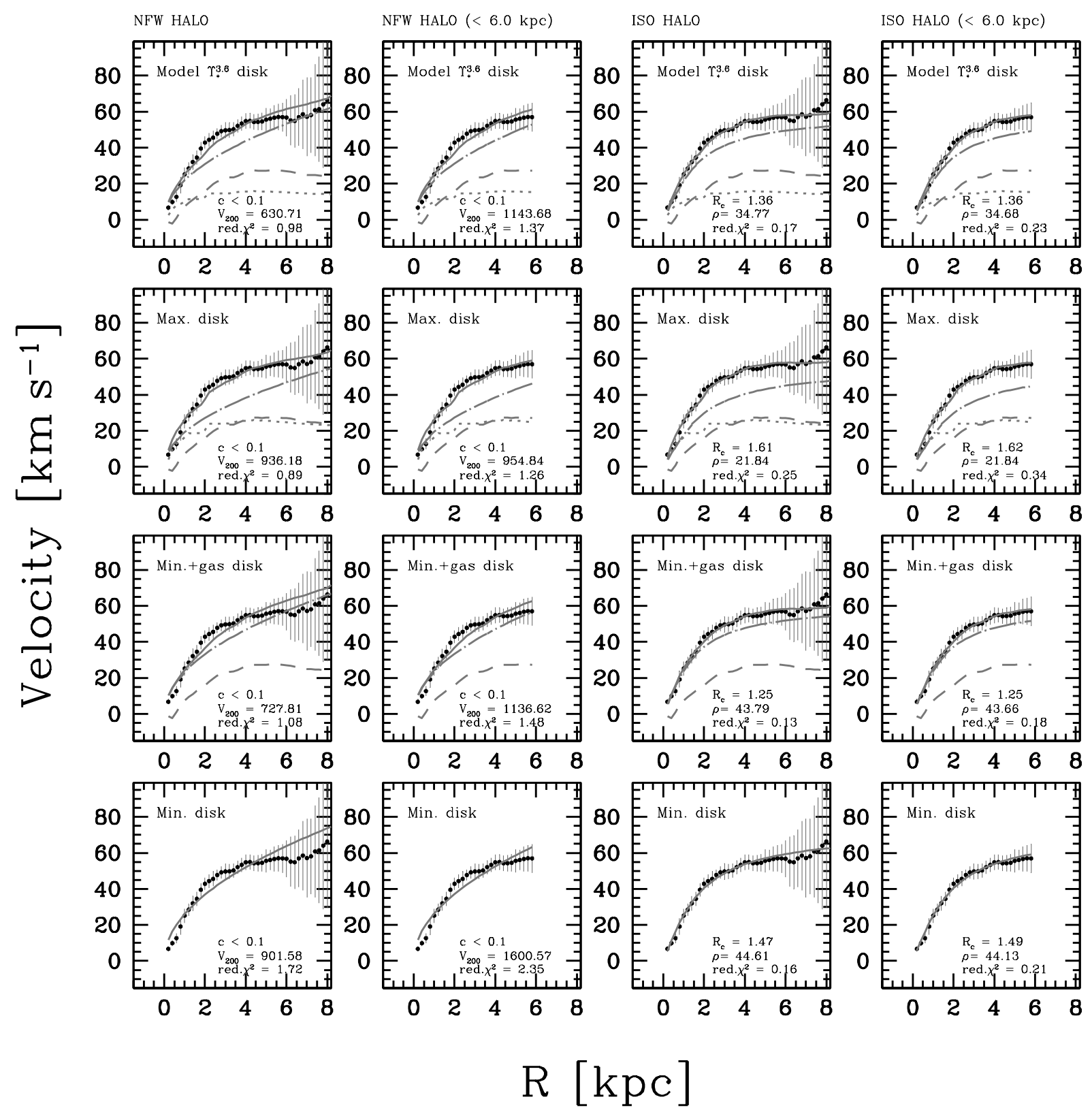

Fig. 22. - Disk-halo decomposition of the NGC 2366 rotation curve under various $\Upsilon_{\star}$ assumptions (model $\Upsilon_{\star}^{3.6}$, maximum disk, minimum disk + gas, and minimum disk). The long dashed lines represent the rotation curves of the gas component; the short dashed lines are the rotation curves of the stellar disk; the long dash-dotted lines the rotation curves of the dark matter halo. The dots are the observed rotation curves from the bulk velocity field while the full lines are the sum of all contributions. The fitted parameters for each halo model (NFW and pseudo-isothermal dark matter halo models) are denoted on each panel along with the reduced $\chi^{2}$ value. Note that the pseudo-isothermal halo gives much better fits to the observed curves than the NFW halo. This is discussed in detail in Section 5.2. 


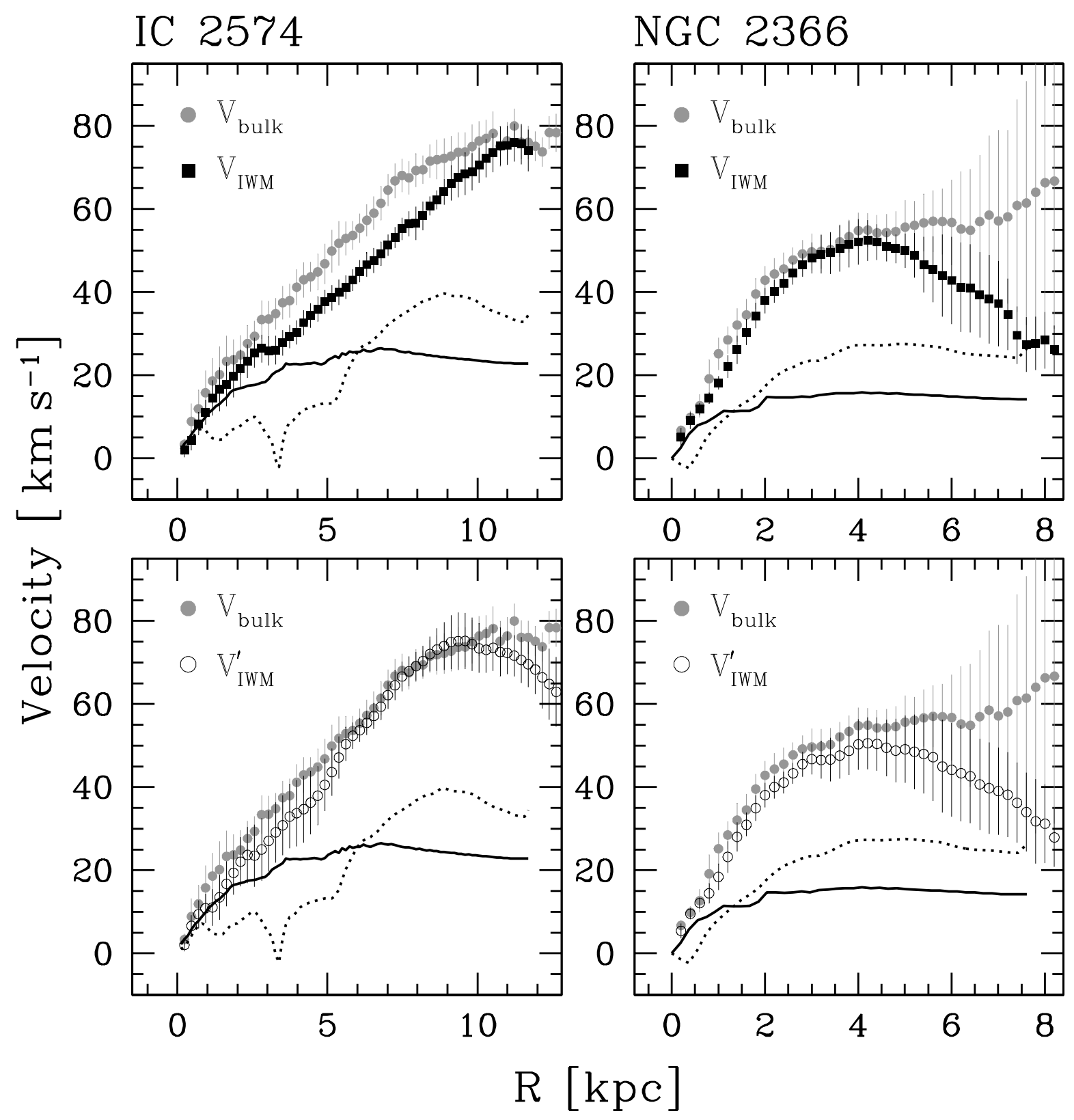

Fig. 23. - Plots showing how non-circular motions affect the mass modeling for IC 2574 and NGC 2366. Filled gray circles represent $V_{\text {bulk }}$. Filled squares and open circles represent $V_{\text {IWM }}$ and $V_{\text {IWM }}^{\prime}$ respectively. The full lines are the contribution to the rotation curves of the stellar component; dotted lines represent the contribution to the rotation curves of the gas component. The derived rotation velocity from the IWM velocity field, $V_{\mathrm{IWM}}$, which is disturbed by non-circular motions, is underestimated. This results in the decreased contribution of dark matter to the observed kinematics since the stellar and gas components remain unchanged. This is discussed in Section 5.2 . 

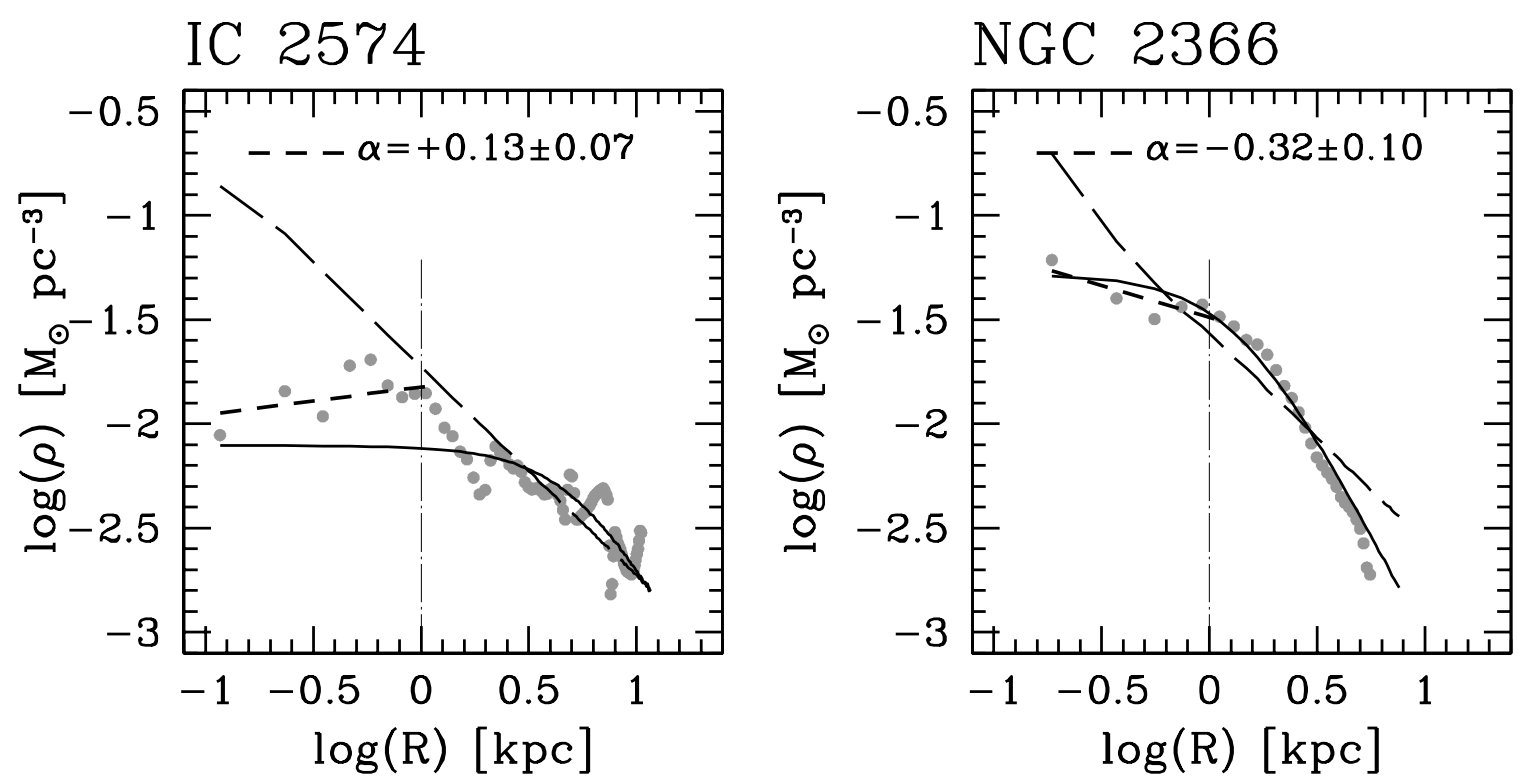

Fig. 24.- The derived mass density profiles of IC 2574 and NGC 2366. Long dashed and solid lines show the NFW halo model and the pseudo-isothermal halo model, respectively. Vertical long dash-dotted lines indicate $1 \mathrm{kpc}$ radius. The filled gray circles represent the dark matter density profile derived from the bulk rotation velocity. The inner slope of the derived dark matter density profile is denoted by $\alpha$ and measured by a least squares fit (short dashed lines) to data points at radii less than $1.2 \mathrm{kpc}$. The measured inner slopes of the mass density profiles of IC 2574 and NGC 2366 are shown in the panels. 


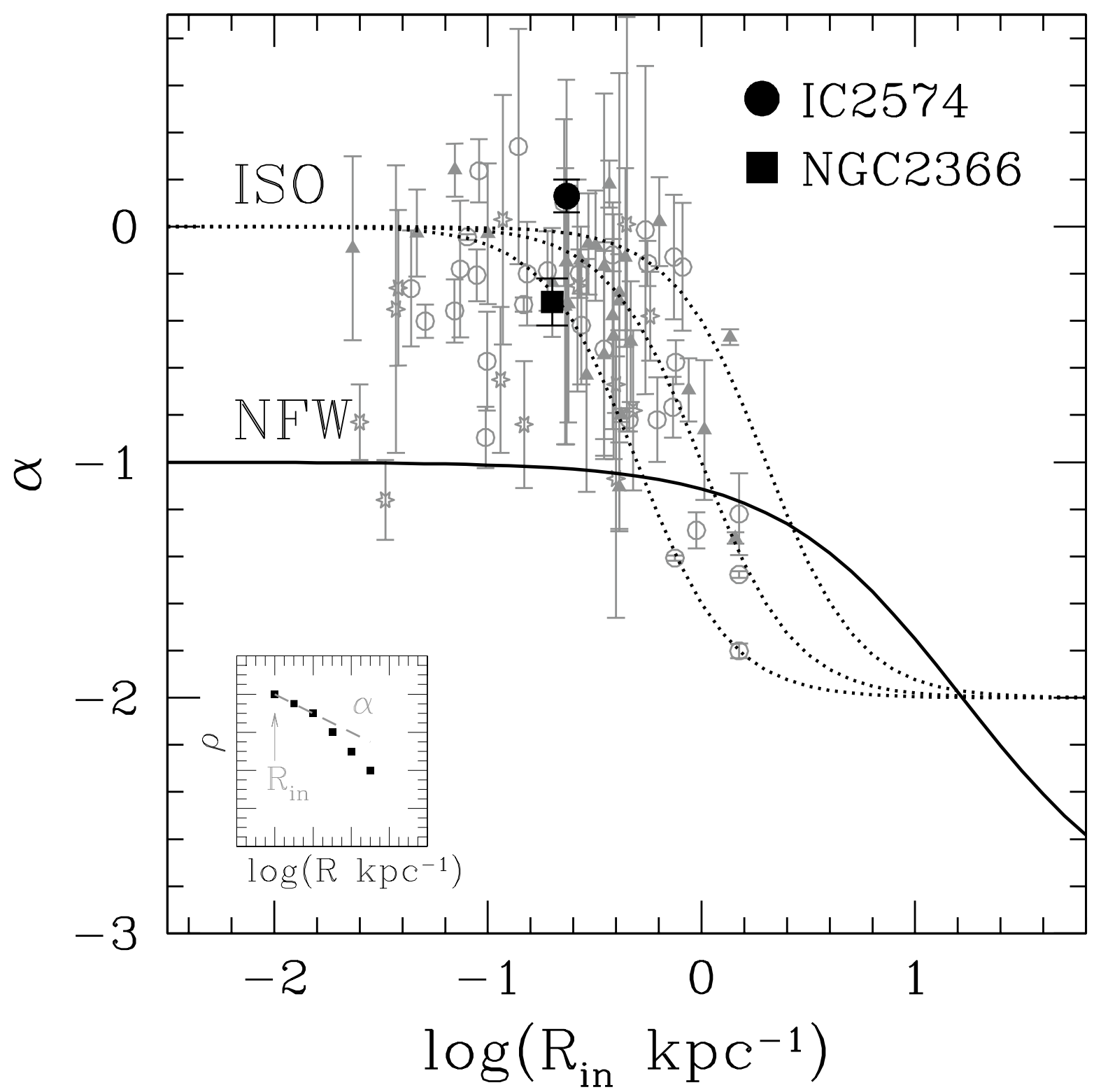

Fig. 25. - The inner slope of the dark matter density profile plotted against the radius of the innermost point. The inner-slopes of the mass density profiles of IC 2574 and NGC 2366 are overplotted with earlier work; they are consistent with previous measurements. Open circles: de Blok et al. (2001); squares: de Blok \& Bosma (2002); open stars: Swaters et al. (2003). The pseudo-isothermal model is preferred over the NFW model to explain the observational data. 